\title{
Lepton number violating operators with standard model gauge fields: a survey of neutrino masses from 3-loops and their link to dark matter
}

\author{
Michael Gustafsson, ${ }^{a}$ José Miguel $\mathrm{No}^{b}$ and Maximiliano A. Rivera ${ }^{c}$ \\ ${ }^{a}$ Institute for theoretical Physics - Faculty of Physics, \\ Georg-August University Göttingen, Friedrich-Hund-Platz 1, D-37077 Göttingen, Germany \\ ${ }^{b}$ Departamento de Fúsica Teórica and Instituto de Física Teórica, \\ IFT-UAM/CSIC, Cantoblanco, 28049, Madrid, Spain \\ ${ }^{c}$ Departamento de Física, Universidad Técnica Federico Santa María, \\ Casilla 110-V, Valparaiso, Chile \\ E-mail: michael.gustafsson@theorie.physik.uni-goettingen.de, \\ josemiguel.no@uam.es, maximiliano.rivera@usm.cl
}

ABSTRACT: We investigate neutrino mass generation scenarios where the lepton number breaking new physics does not interact with Standard Model (SM) quarks and couples only to the SM right-handed charged lepton chirality. The lowest-order lepton number violating effective operator which describes this framework is a unique dimension nine operator involving SM gauge fields, $\mathcal{O}_{9}$. We find that there are two possible classes of new physics scenarios giving rise to this $\mathcal{O}_{9}$ operator. In these scenarios neutrino masses are induced radiatively via dark matter interactions, linking the dark matter to a natural explanation for the smallness of neutrino masses compared to the electroweak scale. We discuss the phenomenology and existing constraints in the different neutrino mass models within each class. In particular, we analyze the important interplay between neutrino mixing and neutrinoless double $\beta$-decay in order to predict characteristic signatures and disfavour certain scenarios.

Keywords: Beyond Standard Model, Neutrino Physics

ARXIV EPRINT: 2006.13564 


\section{Contents}

1 Introduction 1

2 Decomposing the lepton number breaking operator $\mathcal{O}_{9} \quad 2$

3 UV completions of the $\mathcal{O}_{9}$ operator and their phenomenology $\quad 7$

$\begin{array}{lll}3.1 & \text { Class } 1 & 7\end{array}$

3.1.1 Model 1: inert doublet dark matter (the Cocktail model) 9

3.1.2 Model 2: inert triplet dark matter (the Lollipop model) 12

$\begin{array}{lll}3.2 & \text { Class } 2 & 16\end{array}$

3.2.1 Model 1: $S$ with hypercharge $Y=1 . \mathrm{SU}(2)_{L}$ singlets $\chi$ and $S \quad 16$

3.2.2 Model 2: $S$ with hypercharge $Y=1 . \mathrm{SU}(2)_{L}$ triplets $\chi$ and $S \quad 19$

3.2.3 Models 3: $\chi$ with hypercharge $Y=1 \quad 20$

4 Neutrino mixing and neutrinoless double $\beta$-decay 21

$\begin{array}{lll}4.1 & \text { Class } 1 & 23\end{array}$

$\begin{array}{lll}4.2 & \text { Class } 2 & 24\end{array}$

5 Conclusions $\quad 27$

A Oblique parameters in the inert triplet dark matter model 29

\section{Introduction}

The quest to unravel the mechanism responsible for neutrino masses is a central aspect of neutrino physics, tightly linked to the Dirac or Majorana nature of neutrinos. Under the assumption that neutrinos are Majorana particles, a general classification of the possible lepton-number-violating higher-dimensional operators up to dimension $D=11$ that could cause neutrino mass generation has been carried out in $[1,2]$ (see also $[3,4]$ for a recent discussion in the context of neutrinoless double $\beta$-decay). This classification, aimed at providing a general understanding of new physics breaking lepton number by two units $(\Delta L=2)$, focused on higher-dimensional operators built from Standard Model (SM) quarks, leptons as well as the SM Higgs doublet. This classification has however left-out an entire class of $\Delta L=2$ operators, namely those containing also SM gauge fields, as these operators were initially deemed unable to accommodate a suitable renormalizable completion yielding neutrino masses.

In recent years, counterexamples to the latter argument have been found [5-9] ${ }^{1}$ (see also $[11,12]$ for a recent overview of neutrino mass models which include these counterexamples). These examples have in turn unravelled a very interesting connection between the

\footnotetext{
${ }^{1}$ See also [10] for a brief discussion on such counterexamples triggered by correspondence with the Authors of the present manuscript.
} 
presence of SM gauge field in the higher-dimensional $\Delta L=2$ operator, the chirality of the SM leptons present in the operator and its lowest possible dimension $D$ [7]. In a certain way these cases yield generalizations of the "uniqueness" of the Weinberg operator [13], which contains two left-handed chirality leptons, to alternative lowest-order $\Delta L=2$ operators with no SM quarks that instead have left-right (LR) or right-right (RR) chirality assignments to the SM leptons in the operator, as discussed in [7]. In particular, for lepton number violation involving only SM leptons of right-handed chirality (the RR assignment) the lowest order $\Delta L=2$ effective SM operator is unique ${ }^{2}$ and appears at $D=9[7,14]$, which in this work we label as operator $\mathcal{O}_{9}$. Among the salient features of $\mathcal{O}_{9}$ is the fact that neutrino masses from this lepton number violating operator are generated at the earliest at the 2-loop order, thereby providing a natural explanation for the smallness of neutrino masses.

In this work, we analyse in detail the structure and properties of possible renormalizable completions to the operator $\mathcal{O}_{9}$ in beyond Standard Model (BSM) scenarios. At the same time, we show that for a wide range of renormalizable completions there is a connection between neutrino masses and dark matter (DM), with the DM candidate taking an active part in the mechanism of neutrino mass generation. In such cases, neutrino masses are generated at 3 -loops or higher. ${ }^{3}$ We identify two general classes for renormalizable completions of the $\mathcal{O}_{9}$ operator, discuss the general phenomenological aspects of both classes and provide several examples of specific radiative neutrino mass models (several of them genuinely new) belonging to each class.

The paper is organized as follows: section 2 introduces the unique (dimension $D=9$ ) $\Delta L=2 \mathrm{RR}$ operator $\mathcal{O}_{9}$, and discusses various aspects of it. We then proceed to show how every renormalizable completion of this operator can be categorised into two distinct classes of models. In section 3 the two classes' distinct phenomenological features are described and several explicit model realizations are presented. The interplay of neutrino mixing and neutrinoless double $\beta$-decay within each class is studied and its phenomenological impact is discussed in section 4 , before we conclude in section 5 .

\section{Decomposing the lepton number breaking operator $\mathcal{O}_{9}$}

From an Effective Field Theory (EFT) perspective, Majorana neutrino mass generation is described via non-renormalizable (dimension $D \geq 5$ ) operators of SM fields that break lepton number by two units $(\Delta L=2)$. As outlined in the introduction, a general classification of such $\mathrm{SU}(3)_{C} \times \mathrm{SU}(2)_{L} \times \mathrm{U}(1)_{Y}$ gauge invariant operators, ${ }^{4}$ built from SM quarks, leptons and the SM Higgs doublet, is provided in $[1,2]$ up to dimension $D=11$. However, operators involving SM gauge bosons are not contained in this classification. Here we focus on one such $\Delta L=2$ operator involving SM gauge bosons (see figure 1 (left)), given by

$$
\mathcal{O}_{9} \equiv \overline{\ell_{a R}^{c}} \ell_{b R}\left[\left(D_{\mu} H\right)^{T} i \sigma_{2} H\right]^{2},
$$

\footnotetext{
${ }^{2}$ If SM quark fields are allowed in the $\Delta L=2$ effective operator, there exists another $D=9$ operator with RR chirality assignment for the SM leptons, namely $\overline{d_{R}^{c}} u_{R} \overline{d_{R}^{c}} u_{R} \overline{\ell_{R}^{c}} \ell_{R}[3,4]$.

${ }^{3}$ See [15] instead for an analysis of 3-loop neutrino mass models from the Weinberg operator.

${ }^{4}$ We assume that no gauge symmetries beyond of those of the SM (and under which the SM fields are charged) are present.
} 

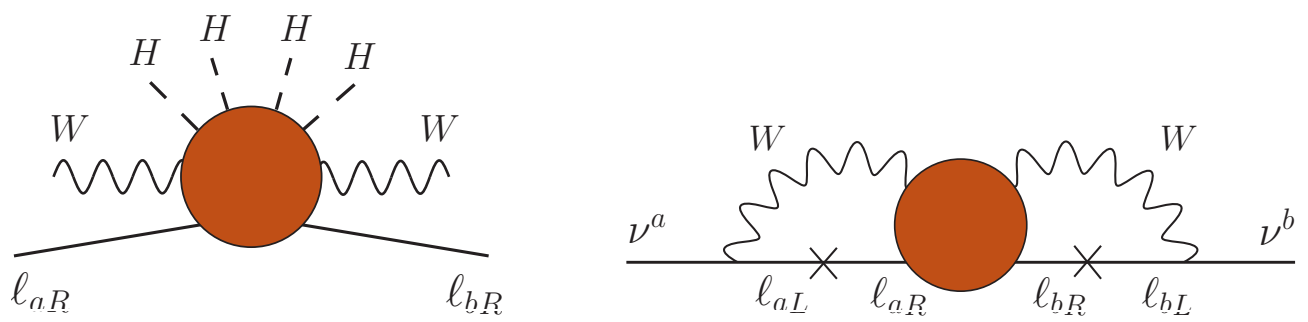

Figure 1. Left: operator $\mathcal{O}_{9}$ from eq. (2.1). Right: 2-loop neutrino mass from $\mathcal{O}_{9}$. The crosses $(\times)$ correspond to SM charged-lepton mass insertions.

with $a, b$ being flavor indices on the right-handed charged leptons $\ell_{R}, H$ being the SM Higgs doublet and $\sigma_{\alpha}(\alpha=1,2,3)$ being the Pauli matrices. Charge conjugation is defined as $\psi^{c} \equiv C\left(\psi^{\dagger}\right)^{T}$, where $C$ includes the proper transformations and group factors for $\psi^{c}$ to transform in the same way as $\psi$ under, in this case, $\mathrm{SU}(2)_{L}$; e.g. $S^{c}=-i \sigma_{2} S^{*}$ for an $\mathrm{SU}(2)_{L}$ doublet scalar $S$, and $\chi^{c}=-i \gamma^{2} \chi^{*}$ for a singlet Dirac spinor $\chi$. This $D=9$ operator is very particular, namely it is the unique lowest order $\Delta L=2$ effective SM operator under the assumption that the new physics breaking lepton number does not couple to SM quarks and couples only to SM leptons of right-handed chirality (and not to those of lefthanded chirality) [7]. Besides its uniqueness, $\mathcal{O}_{9}$ has several features which make it rather appealing for neutrino mass generation: (i) It generates neutrino masses at 2-loop order (see figure 1 (right)), yielding an elegant argument for the smallness of neutrino masses. (ii) In the flavor basis, the entries of the neutrino mass matrix $m_{a b}^{\nu}$ are proportional to the SM charged lepton masses: $m_{a b}^{\nu} \propto m_{a}^{\ell} m_{b}^{\ell}$, which results in specific, testable correlations among neutrino mixing parameters [14]. (iii) It leads to a short-range contribution to neutrinoless double $\beta$-decay processes which dominates over the one from light Majorana neutrino exchange [14], to possibly be within reach of upcoming experiments.

By inspecting figure 1 (left) and eq. (2.1), there are only two possible classes of models that generate the effective operator $\mathcal{O}_{9}$ from a renormalizable theory.

Class 1. The only possible renormalizable interaction involving the charged-lepton bilinear $\overline{\ell_{R}^{c}} \ell_{R}$ involves an $\mathrm{SU}(2)_{L}$ singlet, doubly charged $(Y=2)$ scalar $\rho^{++}[14,16,17]$. The corresponding operator is

$$
C_{a b} \overline{\ell_{a R}^{c}} \ell_{b R} \rho+\text { h.c. }
$$

where $C_{a b}$ is a (complex) Yukawa matrix in flavor space. Trading $\overline{\ell_{R}^{c}} \ell_{R}$ in $\mathcal{O}_{9}$ for $\rho^{c}$ yields the dimension $D=7$ operator [16] (see figure 2 (top-left))

$$
\mathcal{O}_{\mathrm{BSM}}^{1} \equiv \rho^{c}\left[\left(D_{\mu} H\right)^{T} i \sigma_{2} H\right]^{2},
$$

which together with eq. (2.2) breaks lepton number by two units and yields neutrino masses. The neutrino mass matrix reads

$$
m_{a b}^{\nu}=\frac{1}{\left(16 \pi^{2}\right)^{L+2}} C_{a b} \frac{m_{a}^{\ell} m_{b}^{\ell}}{\Lambda},
$$

where $\Lambda$ is an effective energy scale (dependent also on $m_{\rho}$ ) and $L$ is the loop order of the ultraviolet (UV) completion of the operator $\mathcal{O}_{\text {BSM }}^{1}$. Clearly, the operator needs to be 

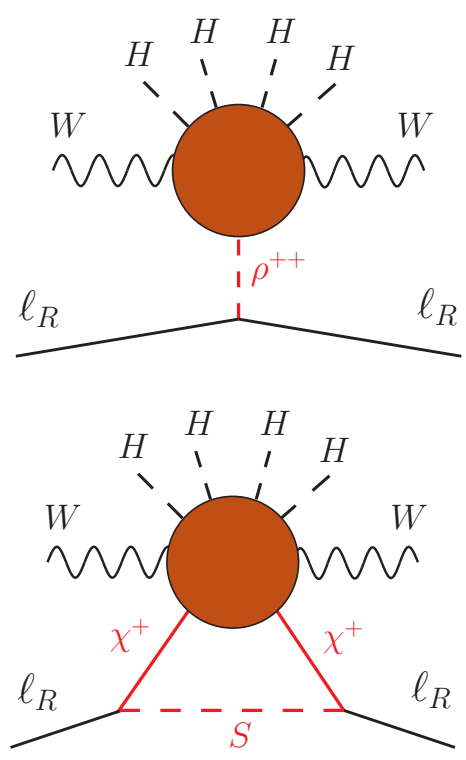
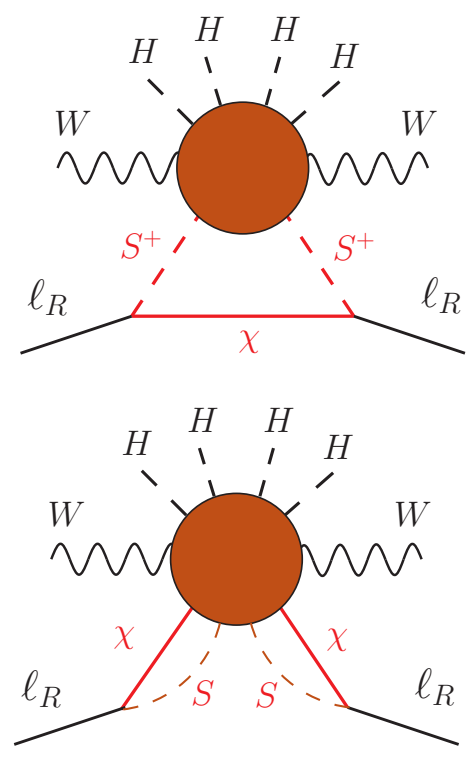

Figure 2. Top-left: opening-up operator $\mathcal{O}_{9}$ in eq. (2.1) via a $\rho^{++}$state, giving rise to the effective BSM operator $\mathcal{O}_{\text {BSM }}^{1}$ of eq. (2.3). Top-right, Bottom-left, Bottom-right: opening-up $\mathcal{O}_{9}$ via $S$ and $\chi$ states, respectively giving rise to the effective BSM operators $\mathcal{O}_{\text {BSM }}^{2 \mathrm{a}}, \mathcal{O}_{\text {BSM }}^{2 \mathrm{~b}}$ and $\mathcal{O}_{\text {BSM }}^{2 \mathrm{c}}$ in eq. (2.6)(2.8). BSM states are depicted in red.

completed as $\rho$ cannot couple directly to $W$ bosons since it is not charged under $\mathrm{SU}(2)_{L}$. Besides, the states (charged under $\left.\mathrm{SU}(2)_{L}\right)$ needed to mediate the interaction between $\rho$ and the $W$ bosons in eq. (2.3) have to lie beyond the SM. The different models within this class then differ in the specific nature and properties of these BSM states. In scenarios without a DM particle candidate, the interactions between $\rho$ and the $W$ bosons can occur at tree-level [5,6], yielding $L=0$ in eq. (2.4). In contrast, for scenarios where the UV completion to $\mathcal{O}_{\text {BSM }}^{1}$ involves DM particles, the operator $\mathcal{O}_{9}$ is generated at 1-loop $[8,9,18]$ (or higher). This can be understood since a DM stabilizing symmetry, e.g. $\mathbb{Z}_{2}$, constrains the allowed interactions to achieve a UV completion, requiring in particular an even number of states charged under the $\mathbb{Z}_{2}$ symmetry. This then yields $L \geq 1$ in eq. (2.4), with DM mediating the interactions between $\rho$ and the $W$ bosons at loop level. As a result, neutrino masses are generated in these scenarios at 3-loops (or beyond).

Class 2. It is also possible to "open-up" the bilinear $\overline{\ell_{R}^{c}} \ell_{R}$ within $\mathcal{O}_{9}$. We introduce a new scalar $S$ and spin 1/2 fermion $\chi$, with the bilinear $\chi S$ being singlet under $\mathrm{SU}(2)_{L}$ and with hypercharge $Y=1$. The SM right-handed charged lepton $\ell_{R}$ can now be coupled to this bilinear via

$$
g_{a}\left(\overline{\chi^{c}} S\right) \ell_{a R}+\text { h.c. }
$$

with $g_{a}$ being a Yukawa coupling. Regarding the hypercharge assignments for $S$ and $\chi$ within the $\chi S$ bilinear, one possibility is for either $\chi$ or $S$ to carry hypercharge $Y=1$ (with the other field having $Y=0$ ). The other possibility is for both $\chi$ and $S$ to have fractional hypercharge (e.g. $Y_{S}=Y_{\chi}=1 / 2$ ). In the former case, this allows to trade the bilinear $\overline{\ell_{R}^{c}} \ell_{R}$ in $\mathcal{O}_{9}$ for either $S^{\dagger} S^{c}$ (if $S$ has hypercharge $Y=1$ ) or $\bar{\chi} \chi^{c}$ (if $\chi$ has 
hypercharge $Y=1$ ), resulting respectively in the effective dimension 8 and 9 operators

$$
\begin{aligned}
& \mathcal{O}_{\mathrm{BSM}}^{2 \mathrm{a}} \equiv S^{\dagger} S^{c}\left[\left(D_{\mu} H\right)^{T} i \sigma_{2} H\right]^{2} \\
& \mathcal{O}_{\mathrm{BSM}}^{2 \mathrm{~b}} \equiv \bar{\chi} \chi^{c}\left[\left(D_{\mu} H\right)^{T} i \sigma_{2} H\right]^{2}
\end{aligned}
$$

as shown in figure 2 (top-right, bottom-left). In the case where $S$ carries hypercharge $Y=1$, in order for lepton number to be broken and neutrino masses to be generated the presence of (2.5) and (2.6) is not enough (lepton number conserving charge assignments for $\chi$ and $S$ exist if only these two terms are present) - a Majorana mass term for the fermion $m_{\chi} \overline{\chi^{c}} \chi$ must also be present. Alternatively, when $\chi$ carries hypercharge $Y=1$ the combined presence of (2.5), (2.7) and a mass term $m_{s}^{2} S^{\dagger} S^{c}$ for the scalar is needed to generate neutrino masses. In addition, the bilinear $\overline{\chi^{c}} S$ in Eq. (2.5) needs to be $S U(2)_{L}$ gauge invariant (together with $S^{\dagger} S^{c}, \bar{\chi} \chi^{c}$ in Eqs. $(2.6,2.7)$ that are manifestly $S U(2)_{L}$ invariant). Both $\chi$ and $S$ then need to transform under the same real representation of $\mathrm{SU}(2)_{L}$. Thus, $\chi$ and $S$ are both $\mathrm{SU}(2)_{L} n$-tuplets with $n$ odd, e.g. both singlets or both triplets. $^{5}$

When $S$ and $\chi$ have fractional hypercharges, the bilinear $\overline{\ell_{R}^{c}} \ell_{R}$ in $\mathcal{O}_{9}$ can only be traded for the $Y=2$ term $\left(\bar{\chi} S^{c}\right)\left(S^{\dagger} \chi^{c}\right)$, yielding the dimension 11 operator (see figure 2 (bottom-right))

$$
\mathcal{O}_{\mathrm{BSM}}^{2 \mathrm{c}} \equiv\left(\bar{\chi} S^{c}\right)\left(S^{\dagger} \chi^{c}\right)\left[\left(D_{\mu} H\right)^{T} i \sigma_{2} H\right]^{2}
$$

which together with (2.5) breaks lepton number by two units. Now only the bilinears $\overline{\chi^{c}} S$ and $\bar{\chi} S^{c}$ need to be $\mathrm{SU}(2)_{L}$ singlets for eqs. (2.5) and (2.8) to be $\mathrm{SU}(2)_{L}$ gauge invariant, which allows $\chi$ and $S$ to transform under pseudoreal representations [19] of $\mathrm{SU}(2)_{L}$ as long as $\chi$ and $S$ transform in the same representation. ${ }^{6}$

The neutrino mass matrix stemming from operators $\mathcal{O}_{\text {BSM }}^{2 \mathrm{a}}$ and $\mathcal{O}_{\mathrm{BSM}}^{2 \mathrm{~b}}$ reads

$$
m_{a b}^{\nu}=\frac{1}{\left(16 \pi^{2}\right)^{L+3}} g_{a} g_{b} \frac{m_{a}^{\ell} m_{b}^{\ell}}{\Lambda^{\prime}},
$$

with $\Lambda^{\prime}$ an effective scale and $L$ the loop order at which the operator $\mathcal{O}_{\text {BSM }}^{2 \mathrm{a}}$ or $\mathcal{O}_{\mathrm{BSM}}^{2 \mathrm{~b}}$ is UV completed by a renormalizable theory of neutrino masses, which requires new fields in addition to $\chi$ and $S$. As shown in figure 2 (top-right, bottom-left) neutrino masses will in this case be generated at three loops or higher. An exception occurs for $\mathcal{O}_{\mathrm{BSM}}^{2 \mathrm{~b}}$ when the neutral component of the field $S$ develops a vev, resulting in $\ell_{R}-\chi$ mixing and neutrino mass generation at two loops or higher [7].

In contrast, for operator $\mathcal{O}_{\mathrm{BSM}}^{2 \mathrm{c}}$ neutrino masses are in general generated at a minimum of four loops, as apparent from figure 2 (bottom-right). The neutrino mass matrix

\footnotetext{
${ }^{5}$ Higher $\mathrm{SU}(2)_{L}$ representations [19] such as quintuplets or septuplets are also possible, yet viable completion of the operators $\mathcal{O}_{\text {BSM }}^{2 \mathrm{a}}, \mathcal{O}_{\mathrm{BSM}}^{2 \mathrm{~b}}$ or $\mathcal{O}_{\mathrm{BSM}}^{2 \mathrm{c}}$ may become phenomenologically more difficult (as shown in e.g. $[20,21])$.

${ }^{6}$ Note that for real representations of $\mathrm{SU}(2)_{L}$, fractional hypercharge $Y=1 / 2$ leads to the existence of new stable particles with fractional electric charge, which is tightly constrained experimentally.
} 


\begin{tabular}{|l|l|l|l|}
\hline Class & Lagrangian & Hypercharges $Y$ & BSM effective operator \\
\hline 1 & $C_{a b} \overline{\ell_{a R}^{c}} \ell_{b R} \rho+$ h.c. & $Y_{\rho}=2$ & $\mathcal{O}_{\mathrm{BSM}}^{1} \equiv \rho^{c}\left[\left(D_{\mu} H\right)^{T} i \sigma_{2} H\right]^{2}$ \\
\hline 2 & $g_{a}\left(\overline{\chi^{c}} S\right) \ell_{a R}+$ h.c. & $Y_{\chi}=0, Y_{S}=1$ & $\mathcal{O}_{\mathrm{BSM}}^{2 \mathrm{a}} \equiv S^{\dagger} S^{c}\left[\left(D_{\mu} H\right)^{T} i \sigma_{2} H\right]^{2}$ \\
& & $Y_{\chi}=1, Y_{S}=0$ & $\mathcal{O}_{\mathrm{BSM}}^{2 \mathrm{~b}} \equiv \bar{\chi} \chi^{c}\left[\left(D_{\mu} H\right)^{T} i \sigma_{2} H\right]^{2}$ \\
& & $Y_{\chi}=q, Y_{S}=1-q$ & $\mathcal{O}_{\mathrm{BSM}}^{2 \mathrm{c}} \equiv\left(\bar{\chi} S^{c}\right)\left(S^{\dagger} \chi^{c}\right)\left[\left(D_{\mu} H\right)^{T} i \sigma_{2} H\right]^{2}$ \\
\hline
\end{tabular}

Table 1. UV completions of the lepton number breaking operator $\mathcal{O}_{9}$ in eq. (2.1) can be divided in two categories (Class 1 and 2) determined by the type of renormalizable BSM interactions with right-handed SM leptons (Lagrangian). Depending on the hypercharge assignments $(Y)$ of the participating BSM fields, the interactions in Class 2 have three different lowest-order BSM effective operators $\left(\mathcal{O}_{\mathrm{BSM}}^{2 \mathrm{a}, 2 \mathrm{~b}, 2 \mathrm{c}}\right)$. For $\mathcal{O}_{\mathrm{BSM}}^{2 \mathrm{c}}, q \neq 0,1$. Different $\mathrm{SU}(2)$ representations are possible for the BSM fields $\chi$ and $S$, see main text for details.

then reads

$$
m_{a b}^{\nu}=\frac{1}{\left(16 \pi^{2}\right)^{L+4}} g_{a} g_{b} \frac{m_{a}^{\ell} m_{b}^{\ell}}{\Lambda^{\prime}}
$$

We note that, similarly to the $\mathcal{O}_{\text {BSM }}^{2 b}$ operator, if the field $S$ has a neutral component and it develops a vev, this can result in neutrino masses being generated at two loops or higher $(L+2)$. Apart from this exception, renormalizable models associated to $\mathcal{O}_{\text {BSM }}^{2 \mathrm{c}}$ will in general yield too small neutrino masses (suppressed by four or more loops), and we do not consider them further in this work.

In constructing the $\mathcal{O}_{\mathrm{BSM}}^{2 \mathrm{a}, \mathrm{b}, \mathrm{c}}$ operators in eqs. (2.6)-(2.8), we have followed the minimalistic approach of adding only one new type of vertex between $\ell_{R}$ and BSM particles. However, the two $S$, or the two $\chi$, to the left and right in the diagrams of figure 2 could in principle be different fields (and hence two different vertices). This would lead to a neutrino mass matrix with a different form from eq. (2.9), i.e., $g_{a} g_{b}$ becomes replaced by $g_{a} \tilde{g}_{b}$ to reflect that the left and right vertex with $\ell_{R}$ are no longer the same. ${ }^{7}$ We will not pursue this potential generalization here, but we note that a systematic investigation in ref. [22] revealed only one such possible realization. ${ }^{8}$

We also stress that for the above classes of models to give $\mathcal{O}_{9}$ as the leading lepton number violating operator, no lower order $\Delta L=2$ operators must be generated by the new fields $\chi$ and $S$. which may require further ingredients depending on the specific quantum numbers of $\chi$ and $S$. An appealing possibility is to consider both fields to be odd under a $\mathbb{Z}_{2}$ symmetry. This automatically forbids interactions like e.g. $H \overline{\ell_{L}} \chi$ (for an $\mathrm{SU}(2)_{L}$ singlet $\chi$, which would lead to the generation of the $\Delta L=2$ Weinberg $D=5$ operator [13]) or

\footnotetext{
${ }^{7}$ This would alter the neutrino mass matrix structure discussed in section 4.2 (and in particular eqs. (4.9), $(4.10))$.

${ }^{8}$ In that setup the two $S^{+}$in the top-right diagram of figure 2 are replaced by two $\mathrm{SU}(2)_{L}$ doublets, $S_{1}$ and $S_{2}$, with hypercharge $Y=3 / 2$ and $Y=1 / 2$, respectively, and $\chi=\chi_{R}+\chi_{L}$ is a $\mathrm{SU}(2)_{L}$ doublet Dirac spinor with $Y=-1 / 2$ to generate the effective operator $S_{1}^{\dagger} S_{2}^{c}\left[\left(D_{\mu} H\right)^{T} i \sigma_{2} H\right]^{2}$.
} 

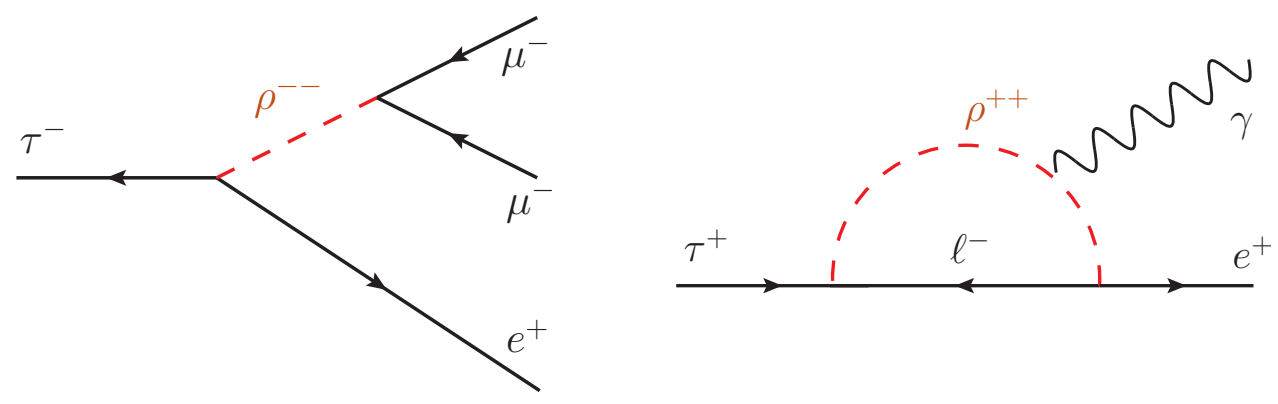

Figure 3. Feynman diagrams in Class 1 for lepton flavour violating processes mediated by $\rho^{ \pm \pm}$at tree-level (Left: $\tau^{-} \rightarrow e^{+} \mu^{-} \mu^{-}$) and loop-level (Right: $\mu^{+} \rightarrow e^{+} \gamma$ ).

$H \bar{\chi} \ell_{R}$ (for an $\mathrm{SU}(2)_{L}$ doublet $\chi$ ). In addition, this $\mathbb{Z}_{2}$ symmetry would stabilize the lightest $\mathbb{Z}_{2}$-odd particle to provide possible DM particle candidates in these scenarios.

This categorization that UV completions of the lepton number violating operator $\mathcal{O}_{9}$ from eq. (2.1) fall into one out of two classes, defined respectively by containing the interaction (2.2) or (2.5), is our first main result and is summarized in table 1 . In the next section we investigate concrete scenarios for neutrino mass generation belonging to Class 1 and Class 2. We discuss the distinct phenomenological features of each class of models, as well as of each individual model within its class.

\section{UV completions of the $\mathcal{O}_{9}$ operator and their phenomenology}

The phenomenology of renormalizable BSM completions of $\mathcal{O}_{9}$ is generically very rich, as a consequence of the required range of masses and couplings of the BSM fields to reproduce the observed pattern of neutrino masses and mixings, and includes potentially measurable lepton flavour violating processes, contributions to electroweak precision observables, signatures at the Large Hadron Collider (LHC) and future colliders, direct and indirect DM signals, as well as short-range contributions to neutrinoless double $\beta$-decay of atomic nuclei. We now discuss general phenomenological aspects as well as specific BSM scenarios for each class, and leave a detailed analysis of the interplay between neutrino mixing and neutrinoless double $\beta$-decay in Class 1 and Class 2 to section 4.

\subsection{Class 1}

The presence of the doubly charged scalar $\rho^{++}$defines the general phenomenology of this class of (radiative) neutrino mass models. We note that when the interactions between $\rho$ and the $W$ bosons in eq. (2.3) occur at tree-level, this is generally through the mixing of $\rho$ with the doubly charged component $\Delta^{++}$of a scalar $\mathrm{SU}(2)_{L}$ triplet $\Delta$ with $Y=1$ (see e.g. $[5,6])$, which affects the properties of $\rho$. In this work we focus instead on the case where the properties of $\rho$ are not altered through its mixing with other states, yielding a loop-induced interaction between $\rho$ and the $W$ bosons.

The presence of the $\rho^{++}$state has a wide impact on phenomenology. First, $\rho$ mediates contributions to various lepton flavour violating processes at tree and loop-level (see e.g. figure 3). In all cases, these contributions scale as $\left(C_{a b} / m_{\rho}\right)^{2}$. For a neutrino mass matrix 


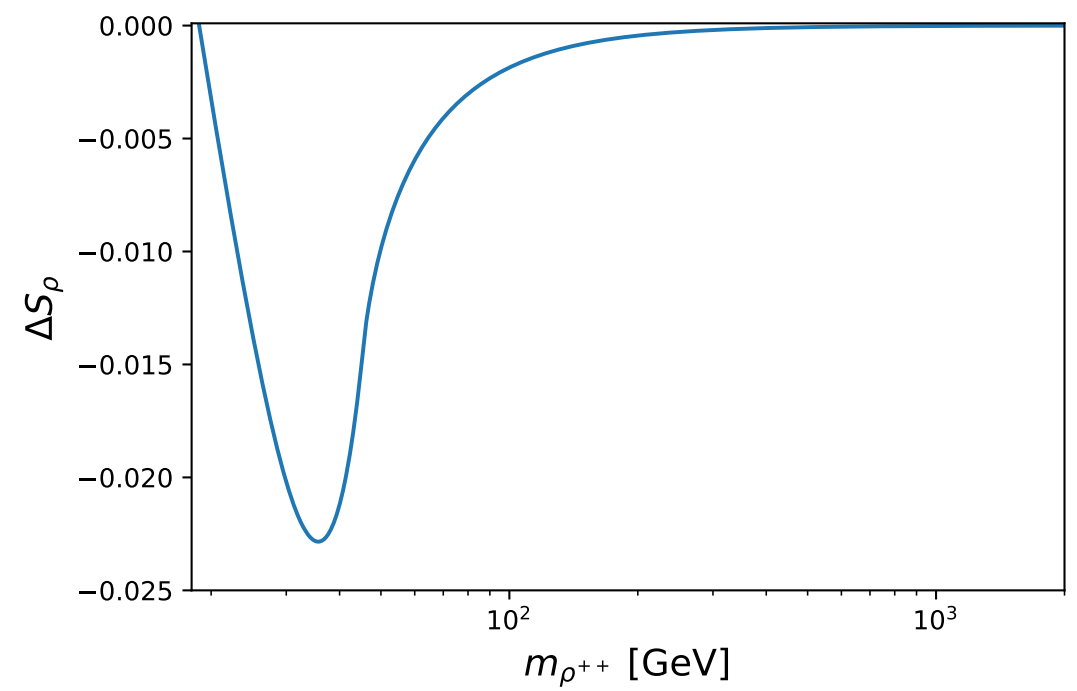

Figure 4. Contribution to the oblique parameter $S$ from the $\mathrm{SU}(2)_{L}$ singlet $\rho^{++}$as a function of its mass $m_{\rho}$. Current experimental result is $S=0.02 \pm 0.10$ [31], with $S=0$ from the SM.

of the form $m_{a b}^{\nu} \propto C_{a b} m_{a}^{\ell} m_{b}^{\ell}$ (as in eq. (2.4)) the matrix entries $m_{e e}^{\nu}$ and $m_{e \mu}^{\nu}$ are in general unavoidably tiny. ${ }^{9}$ Compatibility with the observed pattern of neutrino mixing then requires all other entries in the neutrino mass matrix to be $m_{a b}^{\nu} \sim 0.01 \mathrm{eV}$ (see e.g. figure 4 from [14]). This induces a generic Yukawa hierarchy (see also the discussion in section 4) $C_{e \tau} \gg C_{\mu \mu} \gg C_{\mu \tau} \gg C_{\tau \tau}[14,16]$. As a result, the current most important constraints on the doubly charged scalar $\rho$ from lepton flavour violation are (see e.g. [16, 23-25])

$$
\begin{array}{llll}
\mu^{+} \rightarrow e^{+} \gamma & \mathrm{BR}<4.2 \times 10^{-13}[26] & \rightarrow & \sum_{\ell} C_{\ell \mu} C_{\ell e}^{*} \mid<2.4 \times 10^{-4}\left(m_{\rho} / \mathrm{TeV}\right)^{2} \\
\tau^{-} \rightarrow e^{+} \mu^{-} \mu^{-} & \mathrm{BR}<9.8 \times 10^{-9}[27-29] \rightarrow & \left|C_{e \tau} C_{\mu \mu}\right|<4.1 \times 10^{-3}\left(m_{\rho} / \mathrm{TeV}\right)^{2} \\
\tau^{-} \rightarrow e^{+} e^{-} \mu^{-} & \mathrm{BR}<1.6 \times 10^{-8}[27-29] \rightarrow & \left|C_{e \tau} C_{e \mu}\right|<4.6 \times 10^{-3}\left(m_{\rho} / \mathrm{TeV}\right)^{2} \\
\mu^{-} \rightarrow e^{+} e^{-} e^{-} & \mathrm{BR}<1.0 \times 10^{-12}[30] & \rightarrow & \left|C_{e \mu} C_{e e}\right|<2.3 \times 10^{-5}\left(m_{\rho} / \mathrm{TeV}\right)^{2}
\end{array}
$$

For specific models within Class 1, some of the above lepton flavour violation rates can become large, which generally leads to stringent constraints within these models [25].

The doubly charged scalar $\rho^{++}$contributes to electroweak precision observables through contributions to the oblique parameters $S, T, U$ [32]. Being an $\mathrm{SU}(2)_{L}$ singlet, its contribution to the parameter $T$ vanishes, but not those to the $S$ and $U$ parameters. In figure 4 we show the dependence on the $S$ parameter with the $\rho$ mass, finding that its contribution to the $S$ parameter is well within present experimental constraints. Likewise, $\rho$ 's contribution $U_{\rho}=-S_{\rho}$ [33] remains within the experimental bound $\Delta U \in\{-0.09,0.09\}[31]$. We note that additional contributions to the oblique parameters will come from the fields that UV complete the $\mathcal{O}_{\text {BSM }}^{1}$ operator; these will be discussed below in the context of specific models.

\footnotetext{
${ }^{9}$ The $m_{e}^{2} / v^{2}$ and $m_{e} m_{\mu} / v^{2}$ suppression cannot be compensated by altering any coupling constants or particle masses if they are to be kept within the perturbative and allowed regimes when $L>0$.
} 
Finally, $\rho$ can be searched for at the LHC through Drell-Yan pair production $[17,34,35]$ $p p \rightarrow \gamma^{*}, Z^{*} \rightarrow \rho^{++} \rho^{--}$, with subsequent decays of the doubly charged scalars into dileptons. Decays $\rho^{ \pm \pm} \rightarrow W^{ \pm} W^{ \pm}$are also possible [16, 34-37], as well as cascade decays into BSM states (see e.g. [38]). Regarding the leptonic decays of $\rho^{++}, C_{e \tau}$ is generically the largest entry in the Yukawa matrix $C_{a b}$, and thus the dominant di-leptonic decay mode is $\rho^{ \pm \pm} \rightarrow e^{ \pm} \tau^{ \pm}$in this class of scenarios. This has two important consequences: (i) LHC searches for doubly charged scalars are currently tailored to the decays $\rho^{ \pm \pm} \rightarrow$ $e^{ \pm} e^{ \pm}, e^{ \pm} \mu^{ \pm}, \mu^{ \pm} \mu^{ \pm}[39,40]$. The current limit $m_{\rho}>420 \mathrm{GeV}$ for an $\mathrm{SU}(2)_{L}$-singlet scalar $\rho^{ \pm \pm}[40]$ gets significantly weakened if the decay $\rho^{ \pm \pm} \rightarrow e^{ \pm} \tau^{ \pm}$is dominant, due to the much lower efficiencies for $\tau$-leptons in the final state [35]. Masses down to $m_{\rho} \sim 150 \mathrm{GeV}$ could in principle be allowed. (ii) The dominant decay $\rho^{ \pm \pm} \rightarrow e^{ \pm} \tau^{ \pm}$allows to distinguish this scenario at the LHC and future colliders from others with doubly charged scalars, e.g. the Zee-Babu model [41, 42] where the dominant decay is $\rho^{ \pm \pm} \rightarrow \mu^{ \pm} \mu^{ \pm}$or Type-II see-saw scenarios (see the discussion in [43]).

The collider bounds on $m_{\rho}$ imply that the effective scale $\Lambda$ in eq. (2.4) becomes of similar (or larger) magnitude than such bounds. Sizable Yukawa coupling will then be required to achieve correct neutrino mass matrix entries, e.g. for $m_{e \tau} \simeq 0.01 \mathrm{eV}$ and $\Lambda \simeq 100 \mathrm{GeV}$, eq. (2.4) implies $C_{e \tau} \gtrsim\left(16 \pi^{2}\right)^{(L-1)} \cdot{ }^{10}$ As a result of this combination of unavoidably sizable couplings and not too large masses of BSM states, a rich phenomenology is expected in any particular model realization within this class, with strong constraints on the parameter space arising from the interplay between low and high-energy observables [8, 9, 16, 17, 25]. In the following we discuss in detail two renormalizable models belonging to Class 1 which yield neutrino masses at 3-loops and include a DM particle.

\subsubsection{Model 1: inert doublet dark matter (the Cocktail model)}

This model, originally proposed in [8], contains a scalar $\mathrm{SU}(2)_{L}$ doublet $\Phi$ and a charged scalar $\mathrm{SU}(2)_{L}$ singlet $S^{ \pm}$to complete the $\mathcal{O}_{\mathrm{BSM}}^{1}$ operator of eq. (2.3). Both fields $\Phi$ and $S^{+}$ are assigned to be odd under an unbroken $\mathbb{Z}_{2}$ symmetry, guaranteeing that no SM lepton number violating operator with $D<9$ exists. The SM fields and $\rho^{++}$are even under the $\mathbb{Z}_{2}$ symmetry. The Lagrangian for such a setup reads ${ }^{11}$

$$
\begin{aligned}
\mathcal{L} \supset & \left(D_{\mu} \Phi\right)^{\dagger} D^{\mu} \Phi+\left(D_{\mu} S\right)^{\dagger} D^{\mu} S+\left(D_{\mu} \rho\right)^{\dagger} D^{\mu} \rho-\frac{\lambda_{5}}{2}\left(H^{\dagger} \Phi\right)^{2} \\
& -\kappa_{1} \Phi^{T} i \sigma_{2} H S^{-}-\kappa_{2} \rho^{++} S^{-} S^{-}-\xi \Phi^{T} i \sigma_{2} H S^{+} \rho^{--}-C_{a b} \overline{\ell_{a R}^{c}} \ell_{b R} \rho^{++}+\text {h.c. }
\end{aligned}
$$

where $a, b$ are the flavour indices on the right-handed charged leptons $\ell_{R}$ and $C_{a b}$ is a (complex) Yukawa matrix. The SM Higgs field $H$ and $\Phi$ read

$$
H=\frac{1}{\sqrt{2}}\left(\begin{array}{l}
0 \\
h
\end{array}\right)+\left(\begin{array}{l}
0 \\
v
\end{array}\right) \quad, \quad \Phi=\frac{1}{\sqrt{2}}\left(\begin{array}{c}
\Lambda^{+} \\
H_{0}+i A_{0}
\end{array}\right),
$$

\footnotetext{
${ }^{10}$ Attention might also be required on that perturbativity is ensured in these scenarios.

${ }^{11}$ The additional interactions from the scalar potential $V(H, \Phi, S, \rho)$ are left out for brevity, as they play no significant role in our discussion.
} 


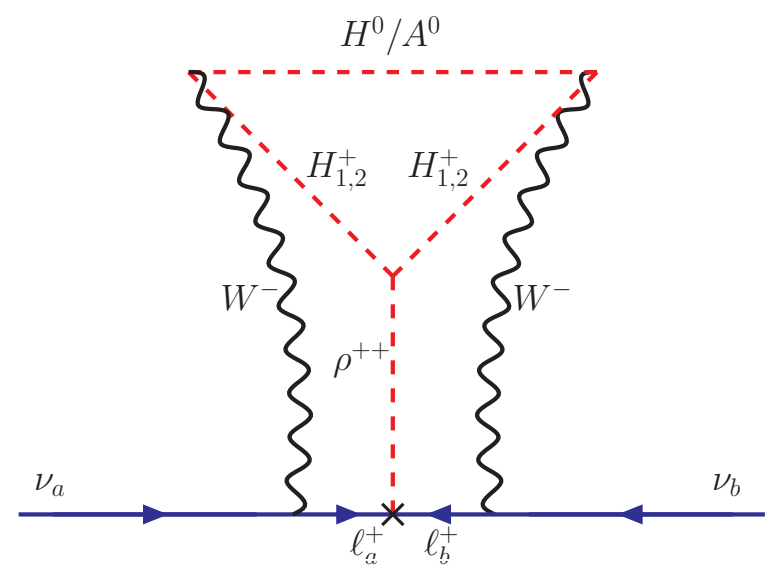

Figure 5. The "cocktail diagram" yielding neutrino masses in the Cocktail model. BSM propagators are depicted in red.

where $v \simeq 174 \mathrm{GeV}$ is the Higgs vacuum expectation value. $H$ and $\Phi$ will not mix due to their different $\mathbb{Z}_{2}$ symmetry properties. The lightest $\mathbb{Z}_{2}$-odd state is stable, and if this is one of the two neutral states $A_{0}, H_{0}$ then the model has a viable DM candidate. In the following we will consider $H_{0}$ to be the DM particle.

After electroweak symmetry breaking the charged states $\Lambda^{+}$and $S^{+}$will mix if $\kappa_{1} \neq 0$ to give the mass eigenstates

$$
\begin{aligned}
& H_{1}^{+}=S^{+} \sin \beta+\Lambda^{+} \cos \beta \\
& H_{2}^{+}=S^{+} \cos \beta-\Lambda^{+} \sin \beta .
\end{aligned}
$$

with the mixing angle being $\beta$. A convenient set of independent parameters for the model are the masses for the five states $\rho, H_{0}, A_{0}, H_{1,2}^{ \pm}$, the mixing angle $\beta$, the couplings $\xi, \kappa_{2}$ and the Yukawa matrix $C_{a b}$. In addition, the remaining parameters of the scalar potential $V(H, \Phi, S, \rho)$ should be properly chosen to preserve vacuum stability (see e.g. [44]) and leave the $\mathbb{Z}_{2}$ symmetry unbroken.

The Lagrangian (3.2) breaks lepton number by two units if both $C_{a b}$ and $\lambda_{5}$ are nonzero together with either $\kappa_{1}, \kappa_{2} \neq 0, \kappa_{1}, \xi \neq 0$ or $\kappa_{2}, \xi \neq 0$. In the first two cases the leading contribution to neutrino masses appears at 3-loop order - via the so-called "cocktail diagram" shown in figure 5 (in contrast, in the latter case the leading contribution to neutrino masses appears at the 5-loop level! This would make the model not phenomenologically viable). The 3-loop neutrino mass matrix $m_{a b}^{\nu}$ has been calculated in $[8,45]$ and is given by

$$
m_{a b}^{\nu}=C_{a b} \frac{m_{a}^{\ell} m_{b}^{\ell} s_{2 \beta}}{\left(16 \pi^{2}\right)^{3} m_{\rho}} \frac{m_{W}^{4} \Delta m_{+}^{2} \Delta m_{0}^{2}}{v^{8}}\left(\mathcal{A}_{1} \mathcal{I}_{1}+\mathcal{A}_{2} \mathcal{I}_{2}\right),
$$

where $m_{a}^{\ell}, m_{b}^{\ell}$ are the SM charged lepton masses (of flavour $\left.a, b\right), s_{2 \beta} \equiv \sin (2 \beta), m_{W}=$ $80.35 \mathrm{GeV}$ is the mass of the $W$-boson, $\Delta m_{+}^{2} \equiv m_{H_{2}^{ \pm}}^{2}-m_{H_{1}^{ \pm}}^{2} \propto \kappa_{1}$ and $\Delta m_{0}^{2} \equiv m_{A_{0}}^{2}-m_{H_{0}}^{2} \propto$ $\lambda_{5}$. The coefficients $\mathcal{A}_{1,2}$ are approximately given by

$$
\mathcal{A}_{1} \simeq \frac{\kappa_{2} s_{2 \beta}+\xi v c_{2 \beta}}{\sqrt{v m_{\rho}}} \quad, \quad \mathcal{A}_{2} \simeq 10 \xi
$$


and the integrals $\mathcal{I}_{1,2}$ are shown to be at most $\mathcal{O}(1)$ numbers when the masses of the new states are kept above current bounds from colliders and couplings are kept in a perturbative regime (see $[8,45]$ for more details).

Besides the presence of the doubly charged scalar $\rho^{++}$, whose most relevant phenomenology has been discussed in section 3.1, the states $H_{1,2}^{+}, A_{0}, H_{0}$ and the connection between neutrino masses and DM yield a wide range of phenomenological implications, which we summarize in the following:

Electroweak precision observables: generating neutrino masses of the right size in the Cocktail model requires large mass splittings among the $\mathbb{Z}_{2}$-odd states $H_{1,2}^{+}, A_{0}, H_{0}$, as $m_{a b}^{\nu}$ is proportional to $\Delta m_{+}^{2}$ and $\Delta m_{0}^{2}$ (see eq. 3.5). These large splittings $\Delta m_{+}^{2}, \Delta m_{0}^{2} \gtrsim v^{2}$ are strongly constrained by measurements of the electroweak oblique parameters, particularly the $T$-parameter (see e.g. [46]). Thus, partial cancellations among different contributions to the oblique parameters are needed in the model. This yields specific mass correlations between the new states [8], $m_{H_{2}^{+}} \gg m_{A_{0}} \gg m_{H_{1}^{+}}, m_{H_{0}}$ (with $m_{H_{2}^{+}}-m_{H_{1}^{+}} \gg v$ and $\left.m_{A_{0}}-m_{H_{0}} \gtrsim v\right)$.

Dark Matter: the DM phenomenology in the Cocktail model is that of the well-studied Inert Doublet model [47-49] (see also [46, 50-54] and references therein). Generating neutrino masses of the right size yields three possible scenarios for obtaining the correct DM relic abundance: (a) $m_{H_{0}} \simeq m_{h} / 2$, the correct DM abundance obtained via annihilation into SM fermions through a resonant SM Higgs boson. (b) $H_{0}-H_{1}^{+}$coannihilations for $m_{H_{0}} \sim 50-75 \mathrm{GeV}$ and $m_{H_{1}^{+}}-m_{H_{0}} \lesssim$ (few) GeV. ${ }^{12}$ (c) $m_{H_{0}} \lesssim m_{W}$, where the closeness to the $W W$ threshold regulates the annihilation rate at freeze-out. All three scenarios remain viable given the current LHC and DM experimental constraints on the model [46, 52-54]. DM direct detection experiments set a strong bound on the DM coupling to the SM Higgs $\lambda_{H_{0}}$, currently $\lambda_{H_{0}} \lesssim 0.03$ for $m_{H_{0}}<100 \mathrm{GeV}$ from the latest XENON1T results [58] (following from an extrapolation of the results in [46]). In addition, the model could produce a striking monochromatic gamma-ray line [59] detectable by the FERMI - Large Area Telescope (see $[53,54]$ for a recent analysis of the Inert Doublet Model in light of indirect DM detection data).

LHC \& future collider phenomenology: the LHC phenomenology of the $\mathbb{Z}_{2}$-odd states $H_{1,2}^{+}, A_{0}, H_{0}$ is similar to that of the Inert Doublet Model, and can lead to multilepton signatures [50] and jet(s) $+E_{T}^{\text {miss }}$ signatures [46, 60,61]. Given the large mass splittings required in the model, the Drell-Yan production processes $p p \rightarrow A_{0} H_{0} \rightarrow H_{0} H_{0} Z, Z \rightarrow \ell \ell$ (mono- $Z$ ), $p p \rightarrow H_{1}^{ \pm} H_{0}$ and $p p \rightarrow H_{1}^{ \pm} H_{1}^{\mp}$ yield the most promising search avenues at the LHC and future collider facilities (we note that jet(s) $+E_{T}^{\text {miss }}$ signatures strongly depend on the value of $\lambda_{H_{0}}$, currently very constrained by DM direct detection). Current constraints from LEP and the LHC are rather weak $[55,56]$ (see also $[46,52]$ ), e.g. the charged state $H_{1}^{+}$is only constrained to lie above $\sim 70 \mathrm{GeV}$. In addition, the presence of $H_{1}^{+}\left(H_{2}^{+}\right.$is

\footnotetext{
${ }^{12}$ This region is constrained by LEP and LHC searches for charginos. A reinterpretation of these limits for the Inert Doublet Model has been performed in [55, 56], yielding $m_{H^{+}} \gtrsim 70 \mathrm{GeV}$. In addition, LHC searches for an invisible width of the $125 \mathrm{GeV}$ Higgs boson yield strong constraints on the $m_{H_{0}}<62 \mathrm{GeV}$ region (see e.g. [57]).
} 
too heavy in general) provides a new possible decay channel $\rho^{ \pm \pm} \rightarrow H_{1}^{ \pm} H_{1}^{ \pm}$, which would yield distinctive high-multiplicity lepton signatures via Drell-Yan pair production of $\rho$ (see e.g. [38]). Finally, the various charged BSM states in the model yield a contribution to $h \rightarrow \gamma \gamma$ (see e.g. [62,63]), which can be important if any of the BSM charged states has a mass around or below $100 \mathrm{GeV}$.

Lepton flavour violation: neutrino masses of the right size within the model require $C_{e \tau} / m_{\rho} \gtrsim 0.1 / \mathrm{TeV}, C_{\mu \mu} / m_{\rho} \gtrsim 10^{-2} / \mathrm{TeV}$ and $C_{\mu \tau} / m_{\rho} \gtrsim 10^{-3} / \mathrm{TeV}$ [8]. As a consequence, the lepton flavour violating processes $\mu \rightarrow e \gamma$ and $\tau^{-} \rightarrow e^{+} \mu^{-} \mu^{-}$strongly constrain the model [25]. By inspecting the various present bounds for lepton flavour violation processes in eq. (3.1), an order of magnitude improvement in current lepton flavour violation sensitivities would then lead to detectable signals or rule out the model.

\subsubsection{Model 2: inert triplet dark matter (the Lollipop model)}

This model has been proposed and analyzed in [9]. Besides the SM fields and the doubly charged scalar $\rho^{++}$, it includes a scalar $\mathrm{SU}(2)_{L}$ triplet $\Delta$ with hypercharge $Y=1$ and a real singlet scalar $\sigma$ in order to enable a UV completion of the $\mathcal{O}_{\text {BSM }}^{1}$ operator in eq. (2.3). As for the previous (Cocktail) model, both fields $\Delta$ and $\sigma$ are odd under an unbroken $\mathbb{Z}_{2}$ symmetry, which guarantees that there is no SM lepton number violating operator with $D<9$. The relevant part of the Lagrangian reads

$$
\begin{aligned}
\mathcal{L} \supset & \operatorname{Tr}\left[\left(D_{\mu} \Delta\right)^{\dagger} D^{\mu} \Delta\right]+\partial_{\mu} \sigma \partial^{\mu} \sigma+\left(D_{\mu} \rho\right)^{\dagger} D^{\mu} \rho-m_{\Delta}^{2} \operatorname{Tr}\left[\Delta^{\dagger} \Delta\right]-\frac{m_{\sigma}^{2}}{2} \sigma^{2} \\
& -\lambda_{H \sigma}|H|^{2} \sigma^{2}-\lambda_{H \Delta}|H|^{2} \operatorname{Tr}\left[\Delta^{\dagger} \Delta\right]-\tilde{\lambda}_{H \Delta} H^{\dagger} \Delta \Delta^{\dagger} H-\kappa_{2} \operatorname{Tr}[\Delta \Delta] \rho^{--} \\
& -\lambda_{6} \sigma H^{\dagger} \Delta \tilde{H}-C_{a b} \overline{\ell_{a R}^{c}} \ell_{b R} \rho^{++}+\text {h.c. },
\end{aligned}
$$

where $H$ is the SM Higgs doublet and $\tilde{H}=i \sigma_{2} H^{*}$ (the additional interactions from the scalar potential $V(\sigma, \rho, H, \Delta)$ are omitted as they will not play a role in the following discussion). The scalar triplet $\Delta$ is given by

$$
\Delta=\frac{1}{\sqrt{2}}\left(\begin{array}{cc}
\Delta^{+} & \sqrt{2} \Delta^{++} \\
\Delta_{0}+i A_{0} & -\Delta^{+}
\end{array}\right) .
$$

After electroweak symmetry breaking the mass hierarchy among $A_{0}, \Delta^{+}$and $\Delta^{++}$is governed by the sign of $\tilde{\lambda}_{H \Delta}$ in eq. (3.7):

$$
m_{A_{0}}^{2}-m_{\Delta^{+}}^{2}=m_{\Delta^{+}}^{2}-m_{\Delta^{++}}^{2}=\tilde{\lambda}_{H \Delta} v^{2} / 2
$$

such that there is either $m_{A_{0}}>m_{\Delta^{+}}>m_{\Delta^{++}}$or $m_{\Delta^{++}}>m_{\Delta^{+}}>m_{A_{0}}$. It can also be seen from eq. (3.7) that lepton number violation in this scenario requires $C_{a b}, \kappa_{2}, \lambda_{6} \neq 0$. After electroweak symmetry breaking, $\lambda_{6}$ induces a mixing between $\Delta_{0}$, the neutral scalar part of the triplet field, and $\sigma$. This singlet-triplet mixing, $\sin \alpha$, yields two mass eigenstates $S_{1}, S_{2}$, with masses

$$
m_{1,2}^{2}=\frac{1}{2}\left[m_{A_{0}}^{2}+\bar{m}_{\sigma}^{2} \pm \sqrt{\left(m_{A_{0}}^{2}-\bar{m}_{\sigma}^{2}\right)^{2}+8 \lambda_{6}^{2} v^{4}}\right]
$$



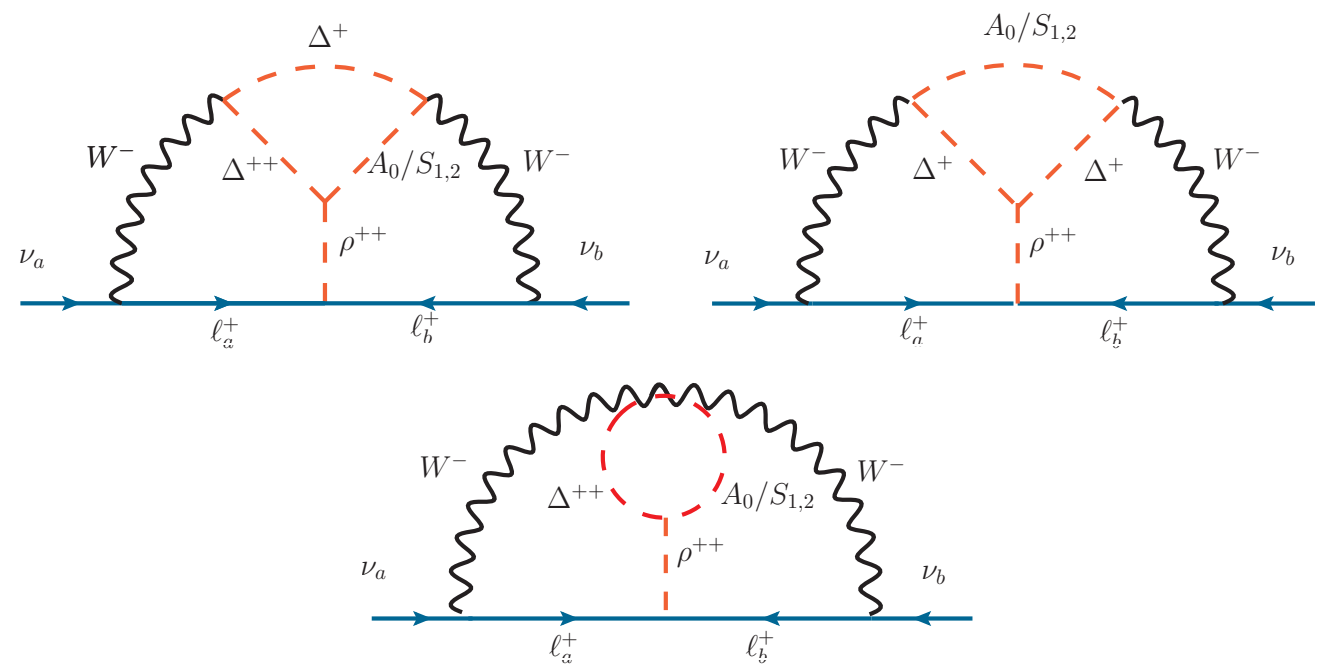

Figure 6. Example Feynman diagrams yielding neutrino masses in the Inert Triplet Dark Matter Model. BSM propagators are depicted in red.

with $\bar{m}_{\sigma}^{2}=m_{\sigma}^{2}+2 \lambda_{H \sigma} v^{2}$. From eq. (3.10) we have $m_{S_{1}}>m_{A_{0}}>m_{S_{2}}$, with the splitting between $A_{0}$ and the mostly triplet-like mass eigenstate (from $S_{1}, S_{2}$ ) being controlled by $\lambda_{6}$. The state $S_{2}$ is the lightest $\mathbb{Z}_{2}$-odd state, and thus a viable DM candidate, provided that $m_{A_{0}}^{2}-m_{S_{2}}^{2}>\tilde{\lambda}_{H \Delta} v^{2}$.

Similarly to the previous scenario, the leading contributions to neutrino masses (shown in figure 6) appear first at 3-loop order. The neutrino mass matrix $m_{a b}^{\nu}$ has been calculated in [9] and is given by

$$
m_{a b}^{\nu}=C_{a b} \frac{m_{a}^{\ell} m_{b}^{\ell}}{\left(16 \pi^{2}\right)^{3} m_{\rho}^{2}} 8 \kappa_{2} \lambda_{6}^{2} \times \mathcal{I}_{\nu}
$$

with $\mathcal{I}_{\nu}$ an integral that takes a value of $\mathcal{O}(1-10)$ provided the new states are close to the electroweak scale [9]. Since the observed neutrino masses and mixing pattern require the entries in the neutrino mass matrix (except for $m_{e e}^{\nu}$ and $m_{e \mu}^{\nu}$ ) to lie in the range $m_{a b}^{\nu} \sim 0.01-0.03 \mathrm{eV}$ (see, e.g. [14]), generating neutrino masses of the right size from eq. (3.11) already requires $\lambda_{6} \gtrsim 1$ and $\kappa_{2} \gtrsim 1 \mathrm{TeV}$. We also note that, if $m_{\rho}$ is to lie below the $\mathrm{TeV}$ scale, $\kappa_{2}$ cannot be pushed much above the $\mathrm{TeV}$ scale since it yields a 1-loop contribution to $m_{\rho}$ of the order $\delta m_{\rho}^{2} \sim \kappa_{2}^{2} /(4 \pi)^{2}$ [42].

The phenomenology of this scenario is also very rich, bearing much resemblance to that of the previous model from section 3.1.1. Both are similar regarding lepton flavour violation, as the doubly charged scalar $\rho^{++}$is the only BSM state interacting with the SM leptons. ${ }^{13}$ However, the DM phenomenology in this scalar triplet DM scenario is notably different from that discussed in section 3.1.1, and the additional presence of the doubly charged scalar $\Delta^{++}$in this model can have important implications for electroweak

\footnotetext{
${ }^{13}$ Yet, since the allowed values of the $\rho^{+}+$mass and its Yukawa couplings to SM leptons may differ from those of the Cocktail model, the predicted strengths of lepton flavour violation processes may be different in the two models.
} 

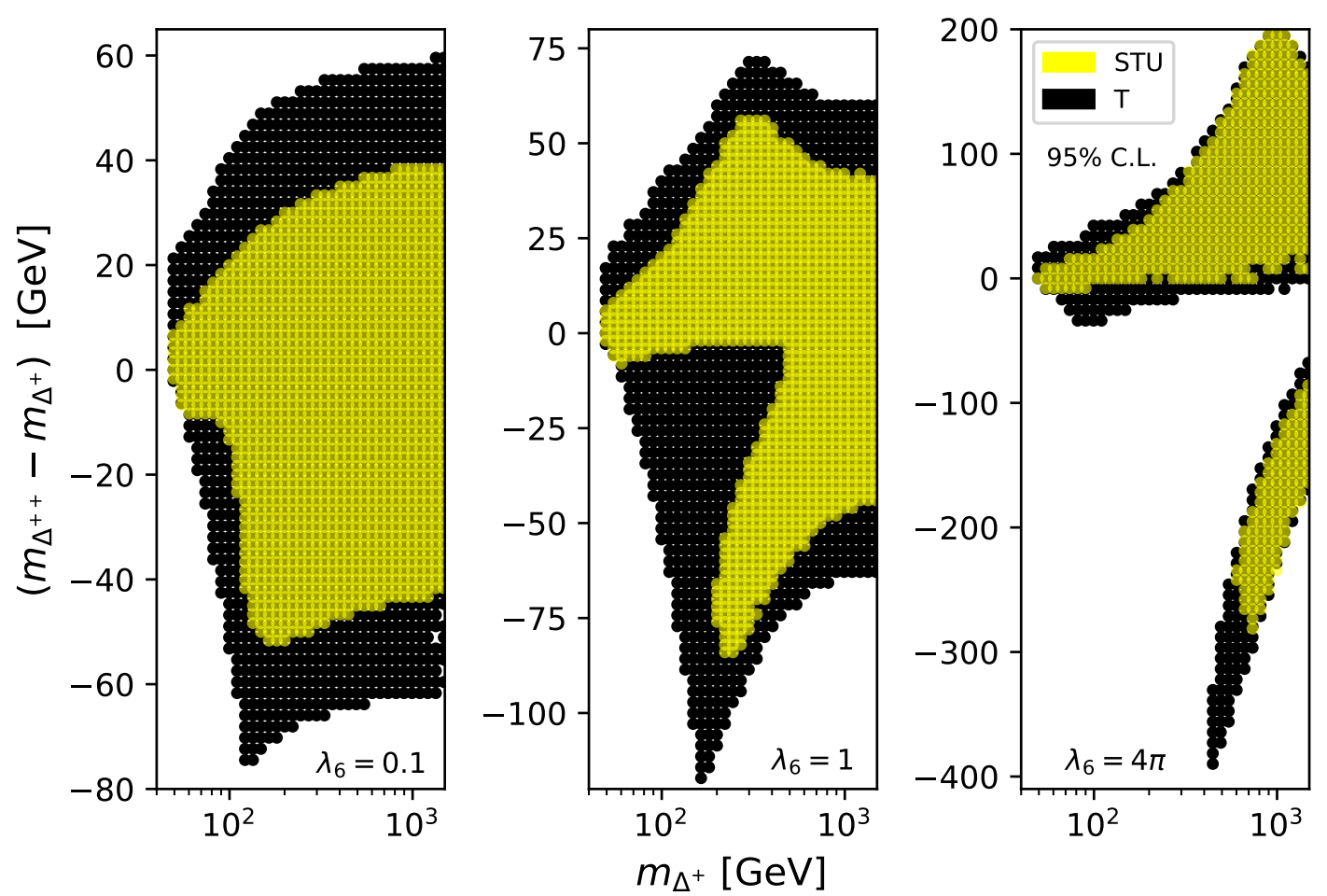

Figure 7. $95 \%$ C.L. allowed regions in the $\left(m_{\Delta^{+}}, m_{\Delta^{++}}-m_{\Delta^{+}}\right)$plane for three different choices of $\lambda_{6}$ (from left to right, $\lambda_{6}=0.1,1,4 \pi$ ) in the Inert Triplet model. Yellow regions consider the combination of $S, T$ and $U$ electroweak precision constraints, while the allowed regions from considering only the $T$ constraint $(\Delta T \in[-0.21,0.27])$ are shown in black.

precision observables and LHC physics. A brief overview of the model's phenomenology is given below.

Electroweak precision observables: besides the $\rho^{++}$contribution to the oblique parameters, which has been discussed above, all the $\mathbb{Z}_{2}$-odd states $\Delta^{+}, \Delta^{++}, A_{0}, S_{1,2}$ yield contributions to the oblique parameter $T$ (as discussed in [9]), as well as to $S$ and $U$. We give explicit expressions for the $S, T$ and $U$ oblique parameters in this model in appendix A. The constraints on $S, T$ and $U$ restricts the allowed mass splittings among the various BSM states. In figure 7 we show allowed model parameter regions under the considered experimental bounds on oblique parameters (see appendix A). Such mass splittings are mainly controlled by the parameters $\tilde{\lambda}_{H \Delta}$ and $\lambda_{6}$, leading e.g. to an allowed splitting $\left|m_{\Delta^{++}}-m_{\Delta^{+}}\right| \lesssim 50 \mathrm{GeV}$ for $\lambda_{6} \lesssim 0.1$ (up to $\left|m_{\Delta^{++}}-m_{\Delta^{+}}\right| \lesssim 300 \mathrm{GeV}$ for $\lambda_{6} \lesssim 4 \pi$ ) at $95 \%$ C.L..

Dark matter: in this scenario $\mathrm{DM}$ is a mixture of the scalar $\mathrm{SU}(2)_{L}$ singlet and the $(Y=1)$ triplet, as guaranteed by $\lambda_{6} \neq 0$. When this DM state $S_{2}$ is dominantly singletlike, the model resembles a Higgs portal singlet DM scenario [64-66] (see also [67, 68] and references therein), and the coupling between the $S_{2}$ state and the SM Higgs is given by

$$
\mathcal{L} \supset-\frac{\lambda_{S_{2}}}{2} S_{2}^{2}\left(2 \sqrt{2} v h+h^{2}\right)
$$


with $\lambda_{S_{2}}=\cos ^{2} \alpha \lambda_{H \sigma}+\sin ^{2} \alpha\left(\lambda_{H \sigma}+\lambda_{H \sigma}\right) / 2-\sqrt{2} \sin \alpha \cos \alpha \lambda_{6}$ being a combination of the various couplings in eq. (3.7) [9]. The DM triplet admixture provides additional (typically dominant) DM annihilation channels once $m_{S_{2}}>m_{W}$. This allows to obtain the correct DM relic density for lower values of $\lambda_{S_{2}}$ as compared to the singlet Higgs portal model, making it easier to satisfy DM direct detection bounds in the present scenario. We also note that $S_{2}-\Delta^{++}$coannihilations are possible when $\tilde{\lambda}_{H \Delta}>0$ and $m_{A_{0}}^{2}-m_{S_{2}}^{2} \sim \tilde{\lambda}_{H \Delta} v^{2}$. It is also worth noting that such a model yields an elegant way to avoid the otherwise extremely stringent direct detection bounds on the DM scattering cross section with nuclei via $Z$-boson exchange which typically rules-out $Y \neq 0$ scalar triplet DM models. ${ }^{14}$

Besides the tree-level contribution to the DM scattering cross section with nuclei, which is mediated by the Higgs boson and scales like $\lambda_{S_{2}}^{2}$, there are important loop-induced DM scattering cross section processes, analogous to those discussed in [69] for the Inert Doublet model. This means that direct detection constraints cannot be completely avoided, even if the freedom in the singlet-triplet mixing ${ }^{15} \sin \alpha$ allows to obtain the correct DM relic density for $\lambda_{S_{2}} \rightarrow 0$. The loop-induced direct detection signal in this model becomes the same as that of [69], but now weighted by $\sin ^{2} \alpha$.

Apart from a potential signal in direct DM detection experiments, the model could yield a monochromatic gamma-ray line [59, 72-76] detectable by the FERMI Large Area Telescope or other gamma-ray telescopes. This signal could be particularly strong for a light $\Delta^{++}$state mediating the DM annihilation into photons. We note that such a signal would be suppressed by $\sin ^{2} \alpha$, but at the same time this mixing needs to be sizable in order to avoid current DM direct detection bounds, as explained above. Thus DM direct and indirect detection could be highly complementary to probe this scenario.

LHC phenomenology: the collider phenomenology of this model bears some resemblance to the one described above for the Cocktail model. For $m_{\Delta^{++}}>m_{\Delta^{+}}>m_{A_{0}}$, the most relevant processes to search for the new scalars at the LHC are those involving the neutral and singly charged states. We note that for sizable values of $\lambda_{6}$ as needed for neutrino masses, $S_{1}$ will be significantly heavier than $S_{2}$. Then, depending on the mass splitting $m_{S_{1}}-m_{S_{2}}$ and the singlet-triplet mixing, either the processes $p p \rightarrow \Delta^{ \pm} S_{1}$, $p p \rightarrow A_{0} S_{1}, p p \rightarrow \Delta^{ \pm} A_{0}$ or $p p \rightarrow \Delta^{ \pm} S_{2}, p p \rightarrow A_{0} S_{2}, p p \rightarrow \Delta^{ \pm} A_{0}$ will yield the main avenues for discovery at the LHC. Both lead to multi-lepton signatures similar to those of the Inert Doublet model discussed before.

In contrast, for $m_{A_{0}}>m_{\Delta^{+}}>m_{\Delta^{++}}$(e.g. in the coannihilation scenario outlined above) the Drell-Yan processes $p p \rightarrow \Delta^{++} \Delta^{--}$and $p p \rightarrow \Delta^{ \pm \pm} \Delta^{\mp}$ may yield the dominant probe of this scenario. In all cases the collider bounds are expected similar to those on the Inert Doublet model, only constraining $\mathcal{O}(100) \mathrm{GeV}$ masses for the new states $[46,52,77,78]$.

\footnotetext{
${ }^{14}$ For an $\mathrm{SU}(2) L$ scalar triplet with $Y=1$, the mass degeneracy between $\Delta_{0}$ and $A_{0}$ states in (3.8) results in a DM scattering cross section with atomic nuclei via $Z$-boson exchange many orders of magnitude above current DM direct detection experimental limits. However, if a mass splitting between $\Delta_{0}$ and $A_{0}$ is induced (here due to the singlet-triplet mixing) the $Z$-boson exchange process will become kinematically forbidden and the model could be experimentally viable.

${ }^{15}$ For the case of singlet-triplet DM models where the scalar triplet instead has $Y=0$, see, e.g. [70, 71].
} 
To conclude this section, we re-emphasize that already for minimal realizations of Class 1 neutrino mass scenarios, like the ones discussed above, the phenomenology that emerges is very rich and all such scenarios share many common phenomenological aspects - ranging from lepton flavour violation to LHC signatures of new charged states. The combination of such various observable aspects serves as a very powerful probe to test these neutrino mass scenarios.

\subsection{Class 2}

The phenomenology of models within this Class 2 of completions of the $\mathcal{O}_{9}$ operator in eq. (2.1) is in principle more diverse than from those in Class 1 discussed above. The reason is that, as discussed in section $2, \chi$ and $S$ can be both either $\mathrm{SU}(2)_{L}$ singlets or triplets, and either $\chi$ or $S$ can be assigned hypercharge $Y=1$. There are however a few general features worth highlighting: (i) As opposed to Class 1 models, there are no lepton flavour violation processes at tree-level in Class 2 models due to the absence of a $\rho^{++}$state. The only important lepton flavour violation process is $\mu \rightarrow e \gamma$, which occurs at 1-loop. (ii) As opposed to Class 1 models, all the BSM states (including $\chi$ and $S$ ) are odd under a $\mathbb{Z}_{2}$ symmetry, which has an important impact on the phenomenology of specific models. (iii) Since the models of Class 2 bear resemblance in particle content (when $S$ carries hypercharge $Y=1$ ) and neutrino mass matrix structure with the KraussNasri-Trodden radiative neutrino mass model [79], the collider phenomenology and search strategies for the BSM states are also quite similar to those used to probe the Krauss-NasriTrodden model and related models [80], in particular regarding multi-leptons [81, 82]. (iv) As already mentioned in section 2, the completion of the operators $\mathcal{O}_{\text {BSM }}^{2 \mathrm{a}}, \mathcal{O}_{\text {BSM }}^{2 \mathrm{~b}}$ in eqs. (2.6) and (2.7) needs in general to occur at tree-level, as otherwise neutrino mass generation happens at 4-loop order, yielding too small neutrino masses to fit neutrino oscillation data. In the following we discuss several renormalizable models belonging to Class 2, corresponding to the states $\chi$ and $S$ being either $\mathrm{SU}(2)_{L}$ singlets or triplets. All these models yield neutrino masses at 3-loops in the presence of DM.

\subsubsection{Model 1: $S$ with hypercharge $Y=1$. SU(2) $L$ singlets $\chi$ and $S$}

This model was introduced in [18] (see also $[83,84]$ ), considering an extension of the SM by two $\mathrm{SU}(2)_{L}$ singlet fermions $\chi_{i} \equiv N_{R_{i}}(i=1,2)$ with $Y=0$, and an $\mathrm{SU}(2)_{L}$ singlet, $Y=1$ scalar $S^{+}$. Here we consider $n>2 \mathrm{SU}(2)_{L}$ singlet fermions (instead of just two) for reasons that we discuss in detail in section 4.2. As argued in section 2, since $S^{+}$is an $\mathrm{SU}(2)_{L}$ singlet we need to introduce extra BSM states in order to mediate its interactions with $W$-bosons, and complete the operator $\mathcal{O}_{\mathrm{BSM}}^{2 \mathrm{a}}$ (see figure 2 (top-right)). A way to do it at tree-level is to also add to the $\mathrm{SM}$ an $\mathrm{SU}(2)_{L}$ scalar triplet with $Y=0[18]$

$$
\Delta=\left(\begin{array}{cc}
\frac{1}{\sqrt{2}} \Delta_{0} & \Delta^{+} \\
\Delta^{-} & -\frac{1}{\sqrt{2}} \Delta_{0}
\end{array}\right) .
$$



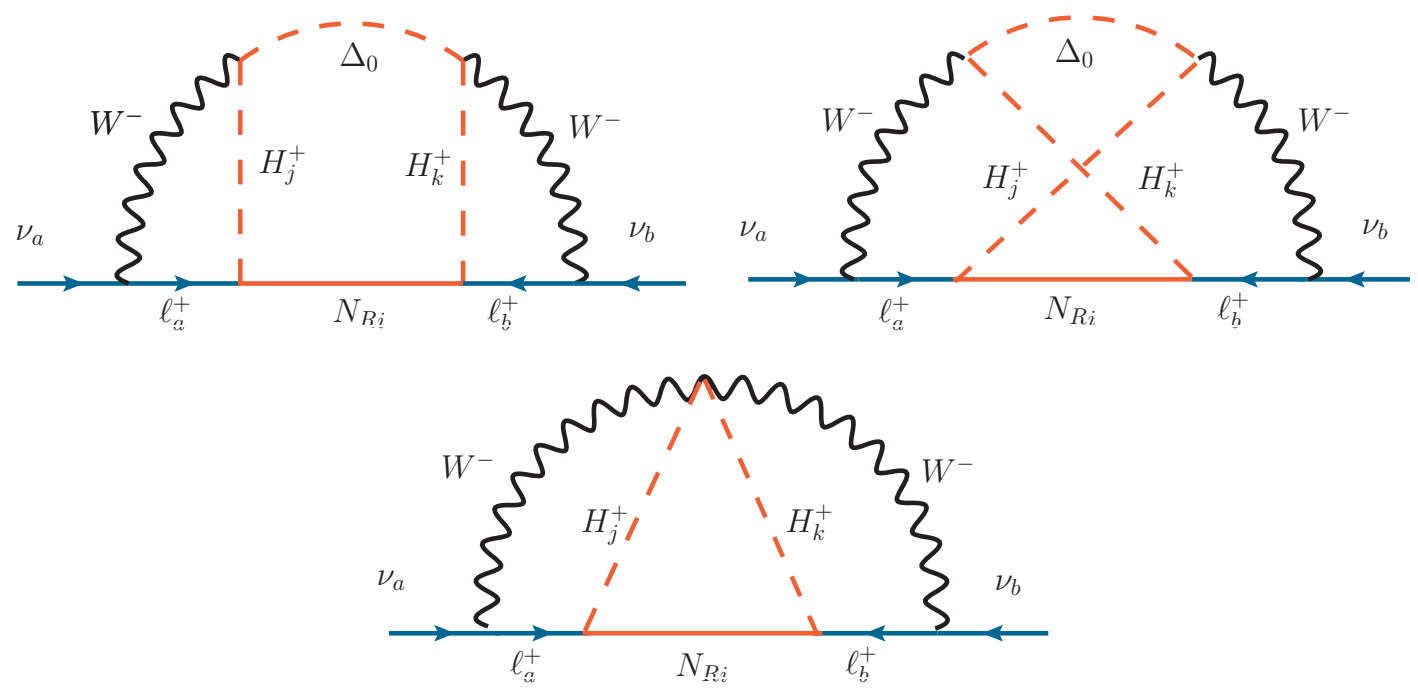

Figure 8. Three-Loop neutrino mass diagrams for Model 1 ( $S$ and $\chi$ being $\mathrm{SU}(2)_{L}$ singlets) within Class 2. BSM propagators are depicted in red.

All the BSM states $\Delta, N_{R_{i}}$ and $S^{+}$are set to be odd under a $\mathbb{Z}_{2}$-symmetry. The Lagrangian is then given by

$$
\begin{aligned}
\mathcal{L}= & \frac{1}{2} \operatorname{Tr}\left[\left(D_{\mu} \Delta\right)^{\dagger}\left(D^{\mu} \Delta\right)\right]+\left(D_{\mu} S\right)^{*}\left(D^{\mu} S\right)+i \overline{N_{R_{i}}} \not \partial N_{R_{i}} \\
& -\frac{1}{2} m_{N_{i}} \overline{N_{R_{i}}} N_{R_{i}}^{c}-g_{i a} \overline{N_{R_{i}}} \ell_{a R^{c}}^{c} S^{-}+\text {h.c. }-V(H, S, \Delta)
\end{aligned}
$$

with $V(H, S, \Delta)$ given by

$$
\begin{aligned}
V(H, S, \Delta)= & -\mu_{H}^{2}|H|^{2}+\mu_{S}^{2}|S|^{2}+\mu_{\Delta}^{2} \operatorname{Tr}\left[\Delta^{2}\right]+\lambda_{H}|H|^{4}+\lambda_{S}|S|^{4} \\
& +\lambda_{\Delta}\left(\operatorname{Tr}\left[\Delta^{2}\right]\right)^{2}+\lambda_{1}|H|^{2}|S|^{2}+\lambda_{2} \operatorname{Tr}\left[\Delta^{2}\right]|S|^{2}+\lambda_{3} \operatorname{Tr}\left[\Delta^{2}\right]|H|^{2} \\
& +\lambda_{4} H^{\dagger} \Delta \tilde{H} S^{+}+\text {h.c. }
\end{aligned}
$$

The combination of $\lambda_{4}$ in (3.15), $g_{i a}$ and $m_{N_{i}}$ in (3.14) breaks lepton number by two units. After electroweak symmetry breaking, $\lambda_{4}$ induces a mixing between $S^{+}$and the charged component of the scalar triplet $\Delta^{+}$. The singlet-triplet mass matrix reads

$$
m^{2}(\Delta, S)=\left(\begin{array}{cc}
2 \mu_{\Delta}^{2}+\lambda_{3} v^{2} \equiv m_{\Delta_{0}}^{2} & \frac{\lambda_{4}}{2} v^{2} \\
\frac{\lambda_{4}}{2} v^{2} & \mu_{S}^{2}+\frac{\lambda_{1}}{2} v^{2}
\end{array}\right)
$$

The singlet-triplet mixing $\sin (\beta)$ gives rise to two charged mass eigenstates $H_{1,2}^{+}$, with

$$
\begin{aligned}
& m_{\Delta_{0}}^{2}=\cos ^{2} \beta m_{H_{1}^{+}}^{2}+\sin ^{2} \beta m_{H_{2}^{+}}^{2} \\
& \lambda_{4} v^{2}=\left(m_{H_{2}^{+}}^{2}-m_{H_{1}^{+}}^{2}\right) \sin (2 \beta)
\end{aligned}
$$

with $\beta \in[0, \pi / 2]$ and we restrict ourselves to $\lambda_{4}>0$ so that $m_{H_{2}^{+}}>m_{\Delta_{0}}>m_{H_{1}^{+}}$. 
The leading contributions to neutrino masses (shown in figure 8) appear at 3-loops. The neutrino mass matrix is given by (see also $[18,83]$ )

$$
m_{a b}^{\nu}=\frac{m_{a}^{\ell} m_{b}^{\ell} \sin ^{2}(2 \beta)\left(m_{H_{1}^{+}}^{2}-m_{H_{2}^{+}}^{2}\right)^{2}}{\left(16 \pi^{2}\right)^{3} v^{4}} \times \sum_{i=1}^{n} \sum_{j=1}^{3} m_{N_{i}} g_{i a} g_{i b} I_{j}\left(m_{N_{i}}\right),
$$

where $I_{j}\left(m_{N_{i}}\right)$ are 3-loop integrals for the three topologies $(j=1,2,3)$ shown in figure 8 .

From eq. (3.17) we observe that there exists an interplay between the generation of sizable neutrino masses, which are proportional to $\left(\lambda_{4} v^{2}\right)^{2}$, and the requirement of satisfying the bounds from electroweak precision observables, in particular from the measurement of the oblique parameter $T$. Its BSM contribution $\Delta T$ is experimentally constrained at $95 \%$ C.L. to the interval (setting in this case $U=0$ ) $\Delta T \in[-0.07,0.17]$ [85], and the contribution of the new scalars is given by

$$
\Delta T=\frac{1}{4 \pi \sin ^{2} \theta_{W} m_{W}^{2}}\left[\cos ^{2} \beta F_{\Delta^{0}, H_{1}^{+}}+\sin ^{2} \beta F_{\Delta^{0}, H_{2}^{+}}-2 \sin ^{2} \beta \cos ^{2} \beta F_{H_{1}^{+}, H_{2}^{+}}\right],
$$

with $\theta_{W}$ the weak mixing angle and

$$
F_{i, j}=\frac{m_{i}^{2}+m_{j}^{2}}{2}-\frac{m_{i}^{2} m_{j}^{2}}{m_{i}^{2}-m_{j}^{2}} \ln \frac{m_{i}^{2}}{m_{j}^{2}} .
$$

For a given value of $\lambda_{4}$, which the neutrino masses depend on directly, there is a minimum value of $m_{H_{2}^{+}}^{2}-m_{H_{1}^{+}}^{2}$ consistent with eq. (3.17), corresponding to a fixed $\lambda_{4} v^{2}$ value. We also find that for fixed $\lambda_{4}$, satisfying the $T$ parameter constraint $\Delta T>-0.07$ imposes a lower bound ${ }^{16}$ on $m_{H_{2}^{+}}^{2}-m_{H_{1}^{+}}^{2}$. This is shown explicitly in figure 9 .

A detailed investigation of the phenomenology of this model and its implications for DM, as well as a comprehensive study of the parameter space for neutrino masses, is left for a forthcoming publication [84]. Regarding the phenomenology of the model, we sketch below the main important aspects to consider:

(i) The collider phenomenology of the model is somewhat similar to that of the KraussNasri-Trodden scenario (as already mentioned above), bearing at the same time much resemblance to leptophilic DM scenarios, with the DM candidate (the lightest of the $N_{R_{i}}$, see below) interacting only with the SM through the SM charged leptons (see e.g. $[86,87]$ for collider analyses of such scenarios). There is however one important difference in the fact that the present model could give rise to vector boson fusion signatures at colliders, since the kinetic term for the scalar triplet field includes the interaction $g^{2} W_{\mu}^{+} W^{\mu+} \Delta^{-} \Delta^{-}+$h.c. $\subset \operatorname{Tr}\left[\left(D_{\mu} \Delta\right)^{\dagger}\left(D^{\mu} \Delta\right)\right]$.

(ii) Concerning lepton flavour violation, the present scenario also resembles the KraussNasri-Trodden model (see e.g. [88]), retaining in our case only the new physics contributions proportional to the right-handed charged lepton couplings $g_{i a}$. The $\mu \rightarrow e \gamma$

\footnotetext{
${ }^{16}$ While this may sound counter-intuitive, bear in mind that for fixed $\lambda_{4}$, a larger mass splitting $m_{H_{2}^{+}}-$ $m_{H_{1}^{+}}$results in a smaller singlet-triplet mixing, which ends up balancing the increase in $m_{H_{2}^{+}}-m_{H_{1}^{+}}$and overall decreasing the value of $|\Delta T|$.
} 


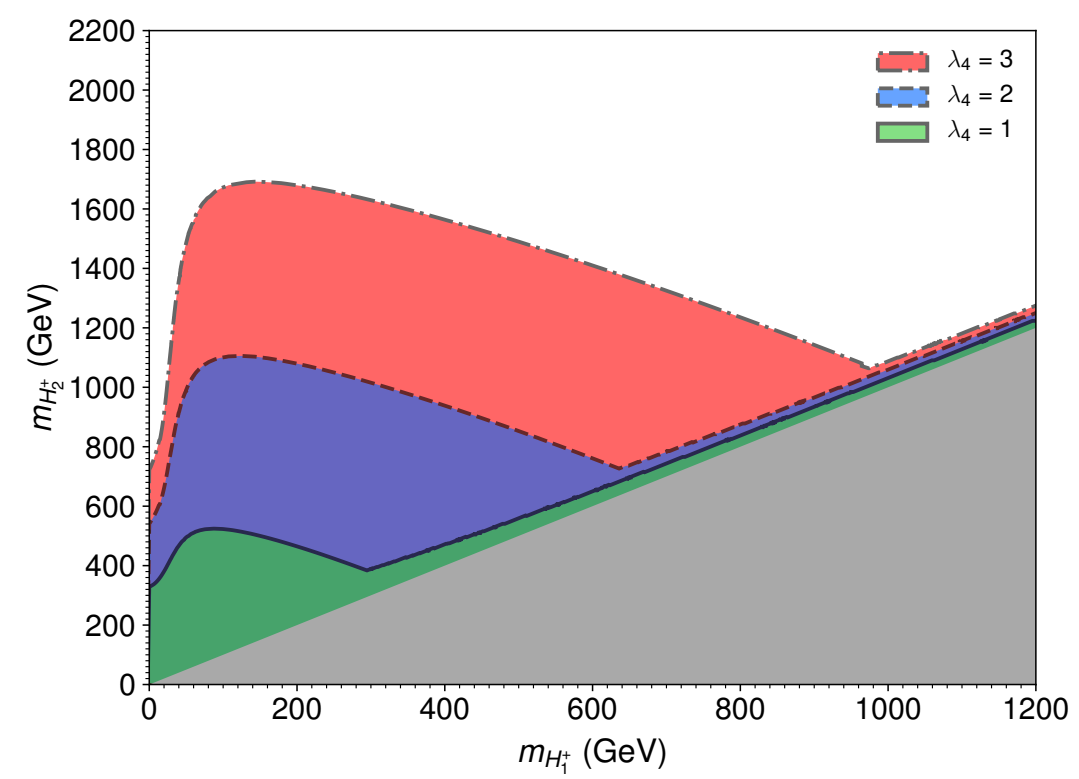

Figure 9. Excluded region (in colour) in the $\left(m_{H_{1}^{+}}, m_{H_{2}^{+}}\right)$plane, for various fixed values of $\lambda_{4}$, from the combination of bounds on electroweak precision observables $(\Delta T \in[-0.07,0.17])$ and eq. (3.17).

process in particular yields stringent constraints on the allowed parameter space of the model [84].

(iii) A viable DM candidate in this model requires the lightest $\mathbb{Z}_{2}$-odd state to be one of the singlet fermions $N_{R_{i}}$ (i.e. the lightest of $N_{R_{i}}$ must be lighter than the charged state $\left.H_{1}^{ \pm}\right)$. We note that the neutral state $\Delta_{0}$ is always heavier than $H_{1}^{ \pm}$and thus cannot be the DM. The DM annihilation in the early Universe is mediated by the Yukawa couplings $g_{i a}$ in eq. (3.14), which generically have to be rather large in order to yield neutrino masses compatible with neutrino oscillation data. This leads to a suppressed DM relic density unless $m_{N_{R_{i}}}$ is correspondingly large, and it is not completely clear if such a setup is phenomenologically viable (e.g. allowing for neutrino masses of the correct size).

\subsubsection{Model 2: $S$ with hypercharge $Y=1$. SU(2) ${ }_{L}$ triplets $\chi$ and $S$}

We now introduce a novel UV completion to the operator $\mathcal{O}_{\text {BSM }}^{2 \mathrm{a}}$ for which the fermions $\chi_{i}$ and scalar $S$ in eq. (2.5) are $\mathrm{SU}(2)_{L}$ triplets. We extend the SM by $n>2 \mathrm{SU}(2)_{L}$ triplet fermions $\chi_{i} \equiv \Sigma_{i}$ with $Y=0$ and an $\mathrm{SU}(2)_{L}$ triplet scalar $S \equiv \Delta$ with hypercharge $Y=1$, and will now use the labels $\Sigma$ and $\Delta$ for our fermion and scalar triplets. Since the state $\Delta$ couples to the SM gauge bosons, we could in principle hope to generate neutrino masses only through the presence of these two BSM fields. Imposing that both fields are odd under a $\mathbb{Z}_{2}$-symmetry, the Lagrangian is

$$
\begin{aligned}
\mathcal{L}= & \frac{1}{2} \operatorname{Tr}\left[\left(D_{\mu} \Delta\right)^{\dagger}\left(D^{\mu} \Delta\right)\right]+i \overline{\Sigma_{R_{i}}} D \Sigma_{R_{i}} \\
& -\frac{1}{2} m_{\Sigma_{i}} \overline{\Sigma_{R_{i}}} \Sigma_{R_{i}}^{c}-g_{i a} \overline{\Sigma_{R_{i}}} \ell_{a R}^{c} \Delta+\text { h.c. }-V(H, \Delta)
\end{aligned}
$$


with $V(H, \Delta)$ depending on even powers of $H$ and $\Delta$. Since $\Delta$ has hypercharge $Y=1$, the Lagrangian (3.21) turns out not to violate lepton number, thus failing to generate neutrino masses. In order to violate lepton number, we further introduce a real singlet scalar field $\sigma$. This introduces (among others) the following additional terms to the Lagrangian

$$
m_{\sigma}^{2} \sigma^{2}+\lambda_{5} \sigma H^{\dagger} \Delta \tilde{H}+\text { h.c. }
$$

which together with the terms in (3.21) break lepton number by two units.

Upon electroweak symmetry breaking, the CP-even neutral component of $\Delta$, which we label $\Delta_{0}$, mixes with the singlet scalar $\sigma$ due to the $\lambda_{5}$ term. When the lightest $\mathbb{Z}_{2}$-odd neutral scalar is lighter than the fermion triplet $\Sigma$, the $\Delta_{0}-\sigma$ mixing makes this scenario viable from the point of view of DM, since DM from a pure inert triplet with $Y=1$ is extremely constrained by DM direct detection experiments, whereas the mixing provides a mass splitting between the states $\Delta_{0}$ and $A_{0}$ (the CP-odd neutral component of $\Delta$ ) within the scalar triplet, thus avoiding the direct detection bounds (recall the discussion in section 3.1.2). Alternatively, when the fermion triplet is lighter than all the $\mathbb{Z}_{2}$-odd scalars, the neutral component of the fermion triplet $\Sigma_{0}$ yields a viable DM candidate. Its relic density is controlled by its gauge interactions together with its interactions with the SM leptons via $g_{1 a}$. Thus, based on the minimal DM predictions (see [89] for more details), a DM mass $m_{\Sigma_{0}} \gtrsim 2.4 \mathrm{TeV}$ is expected in this scenario, with the inequality saturated for $g_{1 a} \ll 1$.

\subsubsection{Models 3: $\chi$ with hypercharge $Y=1$}

As discussed in section 2, it is also possible to generate neutrino masses via the operator $\mathcal{O}_{\text {BSM }}^{2 \mathrm{~b}}$ in (2.7), which together with the interaction (2.5) and the mass term $m_{s}^{2} S^{2}$ break lepton number by two units. In this case, the state $\chi$ has hypercharge $Y=1$ and $S$ has hypercharge $Y=0$, and they can be either $\mathrm{SU}(2)_{L}$ singlets or triplets. In both cases, new physics is required to allow the state $\chi$ to couple to the $\mathrm{SM} \mathrm{SU}(2)_{L}$ gauge bosons in an appropriate way, in order to yield a renormalizable completion of $\mathcal{O}_{\text {BSM }}^{2 b}$.

As we are not aware of any specific neutrino mass model of this type in the literature, we outline in the following two possible setups yielding a renormalizable completion of the $\mathcal{O}_{\text {BSM }}^{2 \mathrm{~b}}$ operator: one with $\chi$ and $S$ being SU $(2)_{L}$ singlets, and then one with $\chi$ and $S$ being $\mathrm{SU}(2)_{L}$ triplets. In both setups we consider $\chi$ and $S$ to be odd under a $\mathbb{Z}_{2}$-symmetry.

When $\chi$ and $S$ are $\mathrm{SU}(2)_{L}$ singlets, the coupling of $\chi$ to the SM gauge bosons may be obtained by introducing an $\mathrm{SU}(2)_{L}$ doublet vector-like ${ }^{17}$ lepton $\Psi$ with hypercharge $Y=1 / 2$ and a Yukawa interaction with $\chi$

$$
Y_{\chi} \bar{\Psi} \tilde{H} \chi+\text { h.c. }
$$

with $H$ the SM Higgs doublet (and $\tilde{H}=i \sigma_{2} H^{*}$ ). Upon electroweak symmetry breaking, the interaction (3.23) leads to a mixing between $\chi$ and the charged component of $\Psi$, which then induces the desired coupling between $\chi$ and the SM gauge bosons. The term (3.23)

\footnotetext{
${ }^{17}$ In order to guarantee that the model remains gauge anomaly-free.
} 
is however not enough to yield lepton number violation in combination with the interaction (2.5) and the mass term $m_{s}^{2} S^{2}$, so some additional ingredient would be required to generate a neutrino mass. We can further add a neutral singlet Majorana fermion $\psi$ with the Lagrangian terms

$$
Y_{\psi} \bar{\Psi} H \psi+m_{\psi} \overline{\psi^{c}} \psi+\text { h.c. . }
$$

The first term yields a mixing between the neutral component of $\Psi$ and the singlet state $\psi$. The combination of (3.23) and (3.24) allows to UV complete the operator $\mathcal{O}_{\mathrm{BSM}}^{2 \mathrm{~b}}$ and give rise to lepton number violation together with (2.5) and the mass term $m_{s}^{2} S^{2}$. Yet, this is achieved at the expense of introducing quite a number of BSM fields.

When $\chi$ and $S$ are instead $\mathrm{SU}(2)_{L}$ triplets, a coupling of $\chi$ to the SM gauge bosons is granted, but it does not directly allow to complete the $\mathcal{O}_{\mathrm{BSM}}^{2 \mathrm{~b}}$ in a renormalizable manner, as the $\chi$ gauge interactions do not lead to lepton number violation. In order to achieve this via direct mixing ${ }^{18}$ with other BSM states, a possibility is again to introduce a vector-like fermion doublet ${ }^{19} \Psi$ with hypercharge $\mathrm{Y}=1 / 2$ and neutral singlet Majorana fermion $\psi$ with the Lagrangian terms

$$
Y_{\chi} \bar{\chi}(H \vec{\sigma} \Psi)+Y_{\psi} \bar{\Psi} H \psi+m_{\psi} \overline{\psi^{c}} \psi+\text { h.c. },
$$

with $\vec{\sigma}$ the Pauli matrices vector and $H \vec{\sigma} \Psi$ transforming as an $\mathrm{SU}(2)_{L}$ triplet fermion with hypercharge $Y=1$. After electroweak symmetry breaking the Majorana singlet fermion $\psi$ mixes with the neutral components of $\Psi$ and $\chi$, allowing for lepton number violation and neutrino mass generation at three-loops. Here also the DM candidate may be the neutral component of the $\mathrm{SU}(2)_{L}$ triplet scalar $S$ (with hypercharge $Y=0$ ), with a phenomenology similar to that of minimal DM [89] up to the effect of the $g_{a}$ coupling to SM leptons from (2.5). As is clear from the discussion in this section, these models are rather cumbersome, and we do not explore them further in this work.

In summary, we find that Class 2 neutrino mass scenarios have been barely explored in the literature, and we leave a more detailed study of specific models for future work [84]. Similarly to Class 1, their phenomenology is expected to be very rich but still somewhat different, in particular regarding lepton flavour violation due to the absence of the BSM state $\rho^{++}$in Class 2 scenarios. In addition, we show in the next section that there are also important differences between Class 1 and Class 2 regarding neutrinoless double $\beta$-decay signatures and their interplay with the pattern of neutrino masses and mixings.

\section{Neutrino mixing and neutrinoless double $\beta$-decay}

We now discuss the interplay between the requirements of fitting the oscillation data for neutrino masses and mixings, and satisfying the bounds from neutrinoless double $\beta$-decay

\footnotetext{
${ }^{18}$ The absence of such a mixing (e.g. lepton number violation via interactions between $\chi$ and other BSM fields, which do not result in a mixing with them) would lead to neutrino masses generated at 4-loop order or beyond.

${ }^{19}$ See [90] for a related implementation of this mechanism for doublet-triplet fermionic DM with hypercharge $Y=0$.
} 
experiments. As we will see below, the combination of these aspects has important consequences for renormalizable completions of the effective operator $\mathcal{O}_{9}$ (this has been studied in some depth for Class 1 models in [14]).

For the case of Majorana neutrinos, a parametrization of their mass matrix, in the basis where charged current interactions are flavour-diagonal and the charged leptons $e, \mu, \tau$ are simultaneously mass eigenstates, reads

$$
m^{\nu}=U^{T} m_{D}^{\nu} U \quad \text { with } \quad m_{D}^{\nu}=\operatorname{Diag}\left(m_{1}, m_{2}, m_{3}\right) .
$$

Here $m_{1,2,3}$ are the masses of the three light neutrinos and $U^{T}$ is the PMNS matrix [91, 92], given in terms of three mixing angles $\theta_{12}, \theta_{23}, \theta_{13}$ and three phases, a CP phase $\delta$ and two Majorana phases $\alpha_{1}$ and $\alpha_{2}$ :

$$
\begin{aligned}
U= & \operatorname{Diag}\left(1, e^{i \alpha_{1}}, e^{i\left(\alpha_{2}+\delta\right)}\right) \\
& \times\left(\begin{array}{ccc}
c_{13} c_{12} & -c_{23} s_{12}-s_{23} c_{12} s_{13} e^{i \delta} & s_{23} s_{12}-c_{23} c_{12} s_{13} e^{i \delta} \\
c_{13} s_{12} & c_{23} c_{12}-s_{23} s_{12} s_{13} e^{i \delta} & -s_{23} c_{12}-c_{23} s_{12} s_{13} e^{i \delta} \\
s_{13} e^{-i \delta} & s_{23} c_{13} & c_{23} c_{13}
\end{array}\right),
\end{aligned}
$$

where $s_{i j} \equiv \sin \left(\theta_{i j}\right)$ and $c_{i j} \equiv \cos \left(\theta_{i j}\right)$. A global fit to the data from different neutrino oscillation experiments gives [93] $\Delta m_{21}^{2} \equiv m_{2}^{2}-m_{1}^{2}=7.39_{-0.20}^{+0.21} \times 10^{-5} \mathrm{eV}^{2},\left|\Delta m_{31}^{2}\right| \equiv$ $\left|m_{3}^{2}-m_{1}^{2}\right|=2.525_{-0.031}^{+0.033} \times 10^{-3} \mathrm{eV}^{2}\left(2.512_{-0.034}^{+0.031} \times 10^{-3} \mathrm{eV}^{2}\right)$ for $\Delta m_{31}^{2}>0\left(\Delta m_{31}^{2}<0\right)$, $s_{12}^{2}=0.310_{-0.012}^{+0.013}, s_{13}^{2}=0.02240_{-0.00066}^{+0.0006}$ and $s_{23}^{2}=0.582_{-0.019}^{+0.015}$ (the atmospheric angle solution in the first octant, $s_{23}^{2} \simeq 0.46$, is currently disfavoured by a bit less than $2 \sigma$ ). Neutrino oscillation experiments are still not fully sensitive to the sign of $\Delta m_{31}^{2}$ which results in two possible mass orderings in the neutrino sector. These are known as normal ordering (NO) and inverted ordering (IO) and are characterized by

$$
\begin{array}{lll}
\Delta m_{31}^{2}>0 & \rightarrow \quad m_{1}<m_{2}<m_{3} \quad(\mathrm{NO}) \\
\Delta m_{31}^{2}<0 & \rightarrow \quad m_{3}<m_{1}<m_{2} \quad(\mathrm{IO}),
\end{array}
$$

with current oscillation data favouring NO over IO at the $2 \sigma-3 \sigma$ level.

Now we turn to look at the neutrinoless double $\beta$-decay probe of Majorana neutrinos. Here we distinguish between two contributions to the neutrinoless double $\beta$-decay process (as shown i figure 10): a long-distance contribution involving light Majorana neutrinos (left panel) and a short-distance contribution that directly involves the lepton number violating effective operator that is responsible for generating neutrino masses, in our case the $\mathcal{O}_{9}$ operator (right panel).

Both the long- and short-distance contributions to neutrinoless double $\beta$-decay are present in general, and which one is dominant depends on the specific type of lepton number violating new physics considered. For the scenarios studied in this work, the operator $\mathcal{O}_{9}$ leads to a neutrino mass matrix at two loops or higher (recall figure 1), with an unavoidably small $m_{e e}^{\nu}$ element, $m_{e e}^{\nu} \ll 10^{-4} \mathrm{eV}$. Thus, the contribution to the neutrinoless double $\beta$ decay amplitude due to a light Majorana neutrino propagator, being proportional to $m_{e e}^{\nu} / p^{2}$ (with $p^{2} \sim(100 \mathrm{MeV})^{2}$ ), is extremely small. However, the $\mathcal{O}_{9}$ operator also gives rise to an effective tree-level short-distance contribution from the diagram shown in the right 

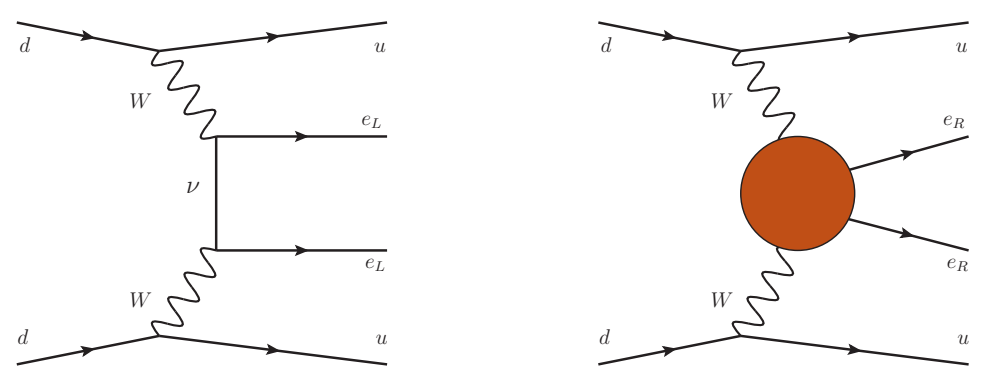

Figure 10. Contributions to the neutrinoless double $\beta$-decay process. Left: light neutrino exchange (long-distance contribution), proportional to $m_{e e}^{\nu}$. Right: short-distance contribution proportional to $\epsilon_{3}$. The solid red blob corresponds to the $\mathcal{O}_{9}$ effective operator.

panel of figure 10, which does not suffer from the extra loop and $\left(m_{e}^{\ell} / v\right)^{2}$ suppression that affects the $m_{e e}^{\nu}$ neutrino mass entry, will largely dominate the neutrinoless double $\beta$-decay amplitude [6, 14].

The emerging effective six-fermion contact interaction from the short-distance contribution corresponding to the right diagram in figure 10 can be written as (see refs. $[94,95]$ )

$$
\mathcal{L}_{0 \nu \beta \beta}=\frac{G_{F}^{2}}{2 m_{p}} \epsilon_{3} J^{\mu} J_{\mu} \bar{e}\left(1-\gamma_{5}\right) e^{c} .
$$

where $J^{\mu}=\bar{u} \gamma^{\mu}\left(1-\gamma_{5}\right) d$ is the vector-axial hadronic current and

$$
\epsilon_{3}=-2 m_{p} \mathcal{A}_{0 \nu \beta \beta}^{\mathrm{SD}},
$$

with $m_{p}$ the proton mass and $\mathcal{A}_{0 \nu \beta \beta}^{\mathrm{SD}}$ the total Feynman amplitude for the short-distance contribution to neutrinoless double $\beta$-decay. The nuclear isotope half-life time $T_{1 / 2}^{0 \nu \beta \beta}$ from neutrinoless double $\beta$-decays due to light neutrino exchange and 1-loop short-distance (SD) interactions are, respectively (see e.g. [96]),

$$
\left[T_{1 / 2}^{0 \nu \beta \beta}\right]^{-1}=g_{A}^{4} G_{01} \frac{\left|m_{e e}^{\nu}\right|^{2}}{\left(m_{e}^{\ell}\right)^{2}}\left|\mathcal{M}^{\nu}\right|^{2} \quad \text { and } \quad\left[T_{1 / 2}^{0 \nu \beta \beta}\right]^{-1}=g_{A}^{4} G_{01}\left|\epsilon_{3}\right|^{2}\left|\mathcal{M}^{\mathrm{SD}}\right|^{2}
$$

Here $g_{A}=1.27$ is the axial vector coupling constant and $G_{01}$ is a nuclear isotope specific phase-space factor while $\mathcal{M}^{\nu}$ (light neutrino exchange) and $\mathcal{M}^{\mathrm{SD}}$ (short-distance) are nuclear matrix elements (NMEs) for the specific isotope. Values on these parameters, together with current experimental limits and future projections for $T_{1 / 2}^{0 \nu \beta \beta}$ for different isotopes can be found in table 2 (for a comprehensive review, see [97]).

The need to fit the neutrino mass and mixing data from oscillation experiments combined with the bounds from neutrinoless double $\beta$-decay affect models within Class 1 and Class 2 in different ways, as we discuss in the following.

\subsection{Class 1}

As presented in section 2, in Class 1 scenarios the electron-electron entry of the Majorana neutrino mass matrix, is given by (recall eq. (2.4))

$$
m_{e e}^{\nu}=\frac{C_{e e}}{\left(16 \pi^{2}\right)^{L+2}}\left(\frac{m_{e}^{\ell}}{v}\right)^{2} \frac{v^{2}}{\Lambda},
$$




\begin{tabular}{|c|c|c|c|c|c|}
\hline & $G_{01}\left(10^{-14} \mathrm{yr}^{-1}\right)$ & $\left|\mathcal{M}^{\nu}\right|$ & $\left|\mathcal{M}^{\mathrm{SD}}\right|$ & $T_{1 / 2}^{0 \nu \beta \beta} \operatorname{limit}\left(\times 10^{26} \mathrm{yr}\right)$ & future $\left(\times 10^{26} \mathrm{yr}\right)$ \\
\hline${ }^{76} \mathrm{Ge}$ & 0.22 & 2.52 & 208.4 & $0.8[98]$ & $10-100[99]$ \\
\hline${ }^{136} \mathrm{Xe}$ & 1.45 & 1.74 & 106.4 & $1.07[100]$ & $20-100[101,102]$ \\
\hline${ }^{130} \mathrm{Te}$ & 1.41 & 2.25 & 192.8 & 0.15 [103] & $>10[104,105]$ \\
\hline
\end{tabular}

Table 2. Values of the phase-space factors $G_{01}$ and nuclear matrix elements $\left|\mathcal{M}^{\nu}\right|$ and $\left|\mathcal{M}^{\mathrm{SD}}\right|$ for different nuclear isotopes. The values of $G_{01}$ are obtained from [96, 106] (see also [107, 108]). The nuclear matrix elements (NMEs) $\left|\mathcal{M}^{\nu}\right|$ are the averaged best estimates from ref. [109]. The NMEs $\left|\mathcal{M}^{\mathrm{SD}}\right|$ are the values given in ref. [110] from their computation using the proton-neutron quasiparticle random phase approximation (pn-QRPA) approach. For ${ }^{76} \mathrm{Ge}$ and ${ }^{136} \mathrm{Xe}$ they are found to agree to better than 30\% with the NME computation from [96] using the interacting shell model (ISM), while for ${ }^{130}$ Te there is roughly a factor two mismatch with ISM. The last two columns show the current best limit on $T_{1 / 2}^{0 \nu \beta \beta}$ for each isotope, as well as the approximate reach of planned experiments for this decade.

is very suppressed, $m_{e e}^{\nu} \ll 10^{-4} \mathrm{eV}$, irrespectively of the value of the Yukawa coupling $C_{e e}$ (as long as it remains perturbative). This approximate neutrino mass texture leads to correlations among neutrino mixing parameters (see [14] for a detailed discussion), via

$$
m_{e e}^{\nu} \equiv c_{13}^{2}\left(m_{1} c_{12}^{2}+e^{2 i \alpha_{1}} m_{2} s_{12}^{2}\right)+e^{2 i \alpha_{2}} m_{3} s_{13}^{2} \sim 0
$$

The same suppression partially affects also the $m_{e \mu}^{\nu}$ entry, proportional to $C_{e \mu}$ and yet to a much lesser extent the $m_{e \tau}^{\nu}$ entry proportional to $C_{e \tau}$. Altogether this leads to several predictions for the ranges of neutrino oscillation parameters, including a NO for neutrino masses, the lightest neutrino mass in the $\sim \mathrm{meV}$ range and a specific correlation between the values of $\theta_{13}$ and $\theta_{23}$ (see [14] for details).

In contrast, for neutrinoless double $\beta$-decay the tree-level short-distance contribution is induced directly by the operator $\mathcal{O}_{\mathrm{BSM}}^{1}$ (which is the core of $\mathcal{O}_{9}$ for Class 1 models), and does not carry the extra two loops and $\left(m_{e}^{\ell} / v\right)^{2}$ suppression that is needed to generate $m_{e e}^{\nu}$. Instead the neutrinoless double $\beta$-decay rates are directly proportional to $C_{e e}$. From lepton flavour violation searches, $C_{e e}$ is constrained not to be too large due to the processes $\rho^{++}$otherwise induce (discussed in section 3.1). Yet, we stress that neutrino oscillation data do not impose restriction on any (perturbative) values of $C_{e e}$.

As a consequence, there is no correlation between the constraints imposed on the parameters of neutrino mass models of Class 1 by neutrino oscillation data, and the predictions for neutrinoless double $\beta$-decay in this models. Experimental bounds on $\epsilon_{3}$ can thus be trivially satisfied since $C_{e e}$ is in essence a free parameter, whose value does not affect neutrino masses and mixings in these models.

\subsection{Class 2}

For Class 2 models, the neutrino mass matrix can be generically written as (recall eq. (2.9))

$$
m_{a b}^{\nu}=\sum_{i=1}^{n} \frac{g_{i a} g_{i b}}{\left(16 \pi^{2}\right)^{L+3}} \frac{m_{a}^{\ell} m_{b}^{\ell}}{v^{2}} \frac{v^{2}}{\Lambda^{\prime}}
$$


with $i=1, \ldots, n$ the number of BSM fermions $\chi_{i}$ in the model. We show in the following that these models face some difficulty in fitting the neutrino oscillation data. For radiative neutrino mass models with BSM fermions $\chi_{i}$ where the flavour structure of the neutrino mass matrix depends on a product of Yukawa couplings, $m_{a b}^{\nu} \propto g_{i a} g_{i b}$, at least $n=2$ is required to fit neutrino oscillation data (as shown in [88] for the particular case of the Krauss-Nasri-Trodden model). Yet, we demonstrate below that $n=2$ is not sufficient for Class 2 models to fit oscillation data.

As for models of Class 1, here the $m_{e e}^{\nu}$ entry of the neutrino mass matrix has to be (for perturbative couplings $\left.g_{i e}\right)$ very small, $m_{e e}^{\nu} \ll 10^{-4} \mathrm{eV}$, due to the $\left(m_{e}^{\ell} / v\right)^{2}$ factor combined with the 3-loop suppression in (4.9). If $n=2$, then the neutrino mass matrix has vanishing determinant, $\operatorname{Det}\left(m^{\nu}\right)=0$, since

$$
m_{a b}^{\nu} \propto\left(\begin{array}{ccc}
g_{1 e} & g_{2 e} & 0 \\
g_{1 \mu} & g_{2 \mu} & 0 \\
g_{1 \tau} & g_{2 \tau} & 0
\end{array}\right)\left(\begin{array}{ccc}
g_{1 e} & g_{1 \mu} & g_{1 \tau} \\
g_{2 e} & g_{2 \mu} & g_{2 \tau} \\
0 & 0 & 0
\end{array}\right)
$$

and as consequence the lightest neutrino is massless. We can then use the dependence of $m_{e e}^{\nu}$ on the neutrino masses and mixings (see eq. (4.8)) to obtain a lower bound on $\left|m_{e e}^{\nu}\right|$ for NO and IO

$$
\begin{aligned}
& \left|m_{e e}^{\nu}\right|>\left|\sqrt{\Delta m_{31}^{2}} s_{13}^{2}-\sqrt{\Delta m_{12}^{2}} s_{12}^{2} c_{13}^{2}\right| \\
& \left|m_{e e}^{\nu}\right|>c_{13}^{2} \sqrt{\Delta m_{13}^{2}}\left|s_{12}^{2}-c_{12}^{2} \sqrt{1-\frac{\Delta m_{21}^{2}}{\Delta m_{13}^{2}}}\right|
\end{aligned}
$$

which altogether yield $\left|m_{e e}^{\nu}\right| \gtrsim 0.001 \mathrm{eV}$, clearly incompatible with the strong $m_{e e}^{\nu}$ suppression discussed above. We note that for the only specific neutrino mass model of Class 2 existing in the literature [18] (see section 3.2.1), $n=2$ was considered, leading to inconsistently large couplings (well beyond the $4 \pi$ perturbativity limit) for the model, as pointed out in [83].

The above problem is however solved for $n \geq 3$, since in this case the lightest neutrino need not be massless and the lower bounds (4.11) do not apply. Yet, for $m_{e e}^{\nu} \ll 10^{-4} \mathrm{eV}$, either $m_{e \tau}^{\nu} \sim m_{\mu \mu}^{\nu} \sim m_{\tau \tau}^{\nu} \sim \sqrt{\left|\Delta m_{31}^{2}\right|}$ or $m_{e \mu}^{\nu} \sim m_{\mu \mu}^{\nu} \sim m_{\tau \tau}^{\nu} \sim \sqrt{\left|\Delta m_{31}^{2}\right|}$ would be approximately required to fit neutrino oscillation data [14]. We focus in the following discussion on $m_{e \tau}^{\nu} \sim \sqrt{\left|\Delta m_{31}^{2}\right|}$, bearing in mind that the discussion of the latter option (with a sizable $m_{e \mu}^{\nu} \sim \sqrt{\left|\Delta m_{31}^{2}\right|}$ ) is analogous in essence but more difficult to be realized phenomenologically. Then, since

$$
m_{a b}^{\nu} \propto \sum_{i=1}^{n} g_{i a} g_{i b} \frac{m_{a}^{\ell} m_{b}^{\ell}}{v},
$$

if we assume no cancellation among the $g_{i a} g_{i b}$ contributions from different $\chi_{i}$ states, $m_{e \tau}^{\nu} \sim$ $m_{\tau \tau}^{\nu}$ leads to $g_{i e} \sim g_{i \tau} \times\left(m_{\tau}^{\ell} / m_{e}^{\ell}\right) \gg g_{i \tau}$ and then we would also have $m_{\tau \tau}^{\nu} \sim m_{e e}^{\nu} \ll 10^{-4} \mathrm{eV}$, again revealing an impossibility to fit neutrino oscillation data. Thus, for $n \geq 3$ there have to exist cancellations among the contributions to (some of) the $m_{a b}^{\nu}$ entries from different $\chi_{i}$ states. This can be illustrated using the model from Class $2 a$ introduced in section 3.2.1 (a 

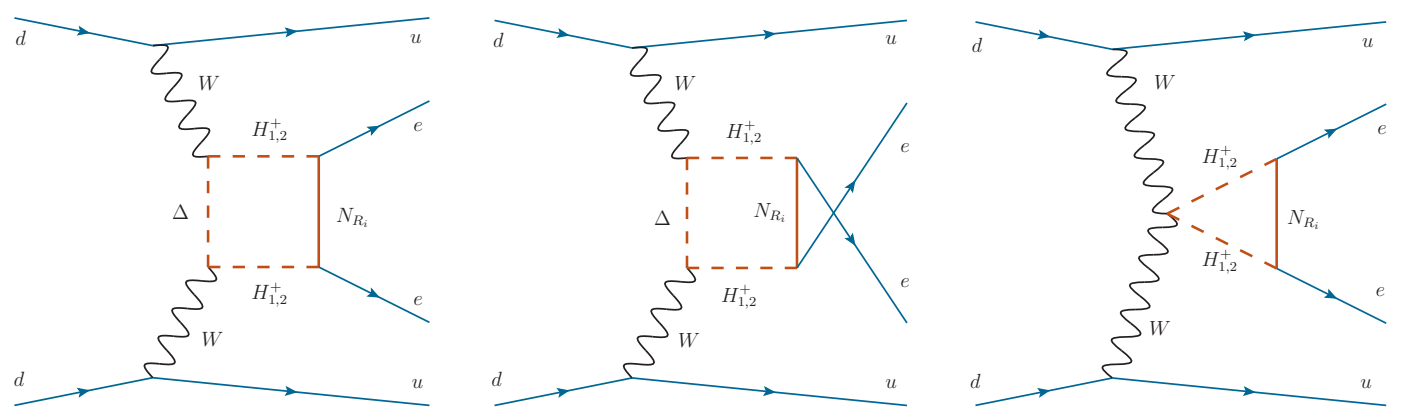

Figure 11. Neutrinoless double $\beta$-decay 1-loop contributions to $\mathcal{A}_{0 \nu \beta \beta}^{\mathrm{SD}}$ for the Class 2 model from section 3.2.1. BSM propagators are depicted in red.

detailed analysis of this model is left for the future [84]), which contains a charged $\mathrm{SU}(2)_{L}$ singlet scalar $S^{+}$and a set of neutral singlet fermions $\chi_{i} \equiv N_{R_{i}}($ with $i=1, \ldots, n ; n \geq 3$ ) as BSM states. The neutrino mass matrix is given in this case by (recall eq. 3.18)

$$
m_{a b}^{\nu}=\lambda_{4}^{2} \times \frac{m_{a}^{\ell} m_{b}^{\ell}}{\left(16 \pi^{2}\right)^{3}} \sum_{i=1}^{n} g_{i a} g_{i b} m_{N_{i}} \sum_{j=1}^{3} I_{j}\left(m_{N_{i}}\right)=\frac{\lambda_{4}^{2} m_{a}^{\ell} m_{b}^{\ell}}{\left(16 \pi^{2}\right)^{3}} \sum_{i} g_{i a} g_{i b} m_{N_{i}} I_{i}
$$

with $I_{j}\left(m_{N_{i}}\right)$ are 3-loop integrals for the three topologies $(j=1,2,3)$ shown in figure 8 , and $\lambda_{4}^{2}$ a dimensionless parameter of the model (see section 3.2.1). Neutrino masses of the right size require $\mathcal{O}(1)$ Yukawa couplings and no cancellation among different $g_{i e} g_{i \tau}$ terms in the $m_{e \tau}^{\nu}$ entry. Then, fitting the observed neutrino mixing pattern from oscillations demands

$$
m_{e \tau}^{\nu} \sim m_{\tau \tau}^{\nu}
$$

which leads to the hierarchy

$$
\sum_{i} g_{i e} g_{i \tau} m_{N_{i}} I_{i} \gg \sum_{i} g_{i \tau} g_{i \tau} m_{N_{i}} I_{i}
$$

for $g_{i e} \sim g_{i \tau} \sim 1$. This clearly implies in general a strong cancellation among different $g_{i \tau} g_{i \tau}$ contributions to $m_{\tau \tau}^{\nu}$.

In addition, there is a further difficulty in simultaneously fitting the neutrino oscillation data and satisfying the constraints from neutrinoless double $\beta$-decay. The model from section 3.2.1 generates 1-loop contributions to the short-distance neutrinoless double $\beta$ decay amplitude $\mathcal{A}_{0 \nu \beta \beta}^{\mathrm{SD}}$, as shown in figure 11 and given by

$$
\mathcal{A}_{0 \nu \beta \beta}^{\mathrm{SD}}=\lambda_{4}^{2} \times \frac{1}{\left(16 \pi^{2}\right)} \sum_{i=1}^{n} g_{i e} g_{i e} m_{N_{i}} \sum_{j=1}^{3} K_{j}\left(m_{N_{i}}\right)=\frac{\lambda_{4}^{2}}{\left(16 \pi^{2}\right)} \sum_{i} g_{i e} g_{i e} m_{N_{i}} K_{i}
$$

where the $K_{j}\left(m_{N_{i}}\right)$ are the separate 1-loop integral contributions related to the topologies shown in figure 11. These contributions are not suppressed by $\left(m_{e}^{\ell} / v\right)^{2}$, as opposed to $m_{e e}^{\nu}$, and for $g_{i e} \sim 1$ the resulting $\mathcal{A}_{0 \nu \beta \beta}^{\mathrm{SD}}$ in fact violates current experimental bounds [83], unless a cancellation among different $g_{i e} g_{i e}$ terms in eq. (4.16) is present. 
It is nevertheless clear that a cancellation in $\mathcal{A}_{0 \nu \beta \beta}^{\mathrm{SD}}$ may be arranged without affecting the model predictions for neutrino masses and mixings. For example, by adding a pair of states $N_{R_{i}}(i=4,5)$ to the model with $n=3$, such that $N_{R_{4,5}}$ only couple to electrons (not to muons or $\tau$-leptons) and demand

$$
\begin{aligned}
& \sum_{i=4}^{5} g_{i e}^{2} m_{N_{i}} K_{i}=-\sum_{i=1}^{3} g_{i e}^{2} m_{N_{i}} K_{i}, \\
& \sum_{i=4}^{5} g_{i e}^{2} m_{N_{i}} I_{i}=0
\end{aligned}
$$

which is a linear system of equations for $g_{4 e}^{2}$ and $g_{5 e}^{2}$ that always has a solution (as long as $K_{4} / I_{4} \neq K_{5} / I_{5}$ ). This leaves $m_{e e}^{\nu}$ (generated by the contributions from $N_{R_{i}}, i=1,2,3$ ) unaffected while yielding a total $\mathcal{A}_{0 \nu \beta \beta}^{\mathrm{SD}}=0$. Still, the introduction of more $N_{R_{i}}$ states with tuned couplings only to cancel the contribution to $\mathcal{A}_{0 \nu \beta \beta}^{\mathrm{SD}}$ seems very much ad hoc, and altogether highlights the generic difficulty of these models to fit neutrino oscillation data and satisfy bounds from neutrinoless double $\beta$-decay, needing in both cases strong tuning among model parameters.

\section{Conclusions}

Among $\Delta L=2$ higher-dimensional SM operators responsible for the generation of neutrino masses, those involving SM gauge fields have so far not been explored in detail in the literature, despite their interesting properties. These include a link between the presence of SM gauge fields and the chirality of the SM charged leptons in the operator, together with an automatic loop suppression of neutrino masses and a sizable contribution to the short-distance neutrinoless double $\beta$-decay amplitude, which generally dominates over the light Majorana neutrino exchange. In this work we have studied in detail the leading of such $\Delta L=2$ operators with two right-handed charged leptons, appearing at $D=9$ and labelled $\mathcal{O}_{9}$ throughout the manuscript. Neutrino masses from this operator are first generated at 2-loop order and further suppressed by the SM charged lepton masses via $m_{a}^{\ell} / v$, thereby providing a natural explanation for their smallness compared to the electroweak scale.

We have analysed the structure and properties of BSM renormalizable completions to the operator $\mathcal{O}_{9}$, finding that there are two possible classes of models for such completions. We have discussed the general features of these completions, highlighting in particular the connection between neutrino masses and DM in these classes of models, with the DM candidate being an integral part of radiative neutrino mass generation. For each class, we have provided examples of specific radiative neutrino mass models (several of them genuinely new), and have discussed the various phenomenological aspects of these models. In table 3 we summarize the very broad phenomenology expected in these scenarios, and highlight "smoking gun" signatures in the two classes which can also enable to phenomenologically distinguish between them. A general feature in both classes is that the leading contribution to neutrino masses appears at 3-loop order with a particular texture $\left(m_{e e}^{\nu} \simeq 0\right.$ and presumably $m_{e \mu}^{\nu} \simeq 0$ as well) of the neutrino mass matrix. This, in turn, leads to well-defined 


\begin{tabular}{|l|c|c|c|c|c|c|c|}
\hline Class & $\begin{array}{c}m_{a b}^{\nu} \\
\text { texture }\end{array}$ & $m_{1}$ & LFV & $0 \nu 2 \beta$ & $\begin{array}{c}\text { DM: } \\
\text { dir. } \mid \text { annh. } \mid \gamma \gamma\end{array}$ & Colliders & EWPO \\
\hline 1 & $\begin{array}{c}\checkmark \\
\left(m_{e e, e \mu}^{\nu} \simeq 0\right)\end{array}$ & $\begin{array}{c}\checkmark \\
(\sim \mathrm{meV})\end{array}$ & $\begin{array}{c}\checkmark \checkmark \\
(\tau \rightarrow e \mu \mu)\end{array}$ & $\bullet$ & $\begin{array}{c}\bullet \mid \\
(W)\end{array}$ & $\begin{array}{c}\checkmark \checkmark \\
(\rho \rightarrow e \tau)\end{array}$ & $\bullet$ \\
\hline 2 & $\begin{array}{c}\checkmark \\
\left(m_{e e, e \mu}^{\nu} \simeq 0\right)\end{array}$ & $\begin{array}{c}\checkmark \\
(\sim \mathrm{meV})\end{array}$ & $\begin{array}{c}\checkmark \checkmark \\
(\mu \rightarrow e \gamma)\end{array}$ & $\begin{array}{c}\checkmark \\
\left(g_{i e}\right)\end{array}$ & $\begin{array}{c}\bullet|\checkmark| \bullet \\
\left(\ell_{R} / W\right)\end{array}$ & $\begin{array}{c}\checkmark \\
(\mathrm{BSM})\end{array}$ & $\bullet$ \\
\hline
\end{tabular}

Table 3. Summary of phenomenological signatures: $(\bullet)$ for potential signals, $(\checkmark)$ for key predictions and $(\checkmark \checkmark)$ for striking signatures specific to Class 1 or Class 2 scenarios. $m_{a b}^{\nu}$ texture: the neutrino mass matrix has a characteristic texture structure in the flavour basis. $m_{1}$ : mass of the lightest neutrino. LFV: characteristic lepton flavour violating processes. $0 \nu 2 \beta$ : neutrinoless double $\beta$-decay signal. DM: dark matter signal in direct detection experiments (dir.), indirect DM annihilation (annh., with dominant annihilation channel) and monochromatic gamma-ray line signature $(\gamma \gamma)$. Colliders: expected generic (BSM) or characteristic signatures. EWPO: electroweak precision observables.

predictions/correlations for certain neutrino mass parameters, e.g. the lightest neutrino mass $m_{1}$ and $\theta_{13}$ (for more details see ref. [14]). Both classes contain a DM particle candidate by construction. For Class 1 models, the multiplet that contains the DM particle interacts directly with the $W$ bosons (see e.g. figure 2 (top-left) and figure 5 ), and indirect DM signatures via DM annihilation into SM gauge bosons are expected in these scenarios. For Class 2 models the DM particle may also couple directly to right-handed SM charged leptons (see e.g. figure 2 (top-right, bottom-left) and figure 8), leading to prominent annihilation into leptons (particularly to $e_{R}$ and $\mu_{R}$ ). In addition, when DM belongs to a heavy $\mathrm{SU}(2)_{L}$ multiplet, a large one-loop annihilation cross section into monochromatic gamma-ray lines can be expected [20, 21, 59, 72-76]. Furthermore, the presence of the doubly charged scalar $\rho^{++}$coupled to the SM charged leptons in Class 1 scenarios gives rise to distinct signatures (not possible in Class 2 models): the (pair) production of $\rho$ at colliders followed by its decay $\rho \rightarrow e \tau$ is a very characteristic signature of these models (due to the sizable $C_{e \tau}$ coupling), together with the lepton flavour violating $\tau$ decay process $\tau^{-} \rightarrow e^{+} \mu^{-} \mu^{-}$. For Class 2 we expect a branching fraction for the lepton flavour violating $\mu \rightarrow e \gamma$ process (potentially not present in Class 1 models) close to the current experimental bound (due to required sizable $g_{i e}$ and $g_{i \mu}$ ). In both Classes, the new states close to the electroweak scale can give rise to a rich variety of collider signatures, yet these will depend on the particular model realization within Class 1 and 2. Similarly, electroweak precision observables constrain masses and mass splittings for new electroweak multiplets, with the potential to give rise to detectable deviations from the SM predictions.

Finally, we have paid special attention to the interplay between neutrino mixing and neutrinoless double $\beta$-decay in these scenarios. Both for Class 1 and Class 2 the dominant contribution to the $\Delta L=2$ neutrinoless double $\beta$-decay process comes from the short-distance amplitude, rather than from the neutrino mass mechanism (light Majorana neutrino exchange). For Class 2 models, the structure of the neutrino mass matrix imposes strong cancellations among parameters in order to fit the neutrino oscillation data, 
and further cancellations in the neutrinoless double $\beta$-decay amplitude parameters $g_{i e}$ are needed to satisfy the current experimental bounds from e.g. KamLAND-Zen (the possible viability of these models given the needed cancellations will be explored in [84]). This is in contrast with Class 1 scenarios, for which no such cancellations are required. Altogether, neutrino mass models from $\mathcal{O}_{9}$ turn out to be very predictive as a result of the many different phenomenological aspects they are linked to, which also result in these models (particularly for Class 2) being severely constrained experimentally.

\section{Acknowledgments}

We thanks Diego Aristizabal for many useful discussions and comments on the manuscript, and Martin Hirsch for comments on an earlier version of this manuscript. We also want to thank Arcadi Santamaria for discussions on radiative neutrino masses and model building, as well as Raymond Volkas for correspondence many years ago which led us to think more seriously about these issues. M.G. acknowledges partial support from the European Unions Horizon 2020 research and innovation program under grant agreement No 690575 and No 674896. M.A.R acknowledges partial support from Anillo grant PIA/ACT1406, CONICYT, Chile; Fondecyt grant 1171136, Chile and Proyecto Interno USM PI.L.18.23 "Phenomenology Of Neutrinos and Dark Matter in Extensions of the Standard model". J.M.N. was supported by Ramón y Cajal Fellowship contract RYC-2017-22986, and also acknowledges support from the Spanish MINECO's "Centro de Excelencia Severo Ochoa" Programme under grant SEV-2016-0597, from the European Union's Horizon 2020 research and innovation programme under the Marie Sklodowska-Curie grant agreements 690575 (RISE InvisiblesPlus) and 860881 (ITN HIDDeN) and from the Spanish Proyectos de I+D de Generación de Conocimiento via grant PGC2018-096646-A-I00.

\section{A Oblique parameters in the inert triplet dark matter model}

In order to analyze the impact on the electroweak precision observables from the inert triplet model in section 3.1.2 (see also [9]), we compute the oblique $S, T$ and $U$ parameters [32]. Following the notation of [111], they read

$$
\begin{aligned}
\frac{\alpha_{\mathrm{em}}}{4 c_{W}^{2} s_{W}^{2}} S & \equiv \frac{\Pi_{Z Z}\left(m_{Z}^{2}\right)-\Pi_{Z Z}(0)}{m_{Z}^{2}}-\frac{c_{W}^{2}-s_{W}^{2}}{c_{W} s_{W}} \Pi_{Z \gamma}^{\prime}(0)-\Pi_{\gamma \gamma}^{\prime}(0), \\
\alpha_{\mathrm{em}} T \equiv & \frac{\Pi_{W W}(0)}{m_{W}^{2}}-\frac{\Pi_{Z Z}(0)}{m_{Z}^{2}}, \\
\frac{\alpha_{\mathrm{em}}}{4 s_{W}^{2}} U \equiv & \frac{\Pi_{W W}\left(m_{W}^{2}\right)-\Pi_{W W}(0)}{m_{W}^{2}}-c_{W}^{2}\left[\frac{\Pi_{Z Z}\left(m_{Z}^{2}\right)-\Pi_{Z Z}(0)}{m_{Z}^{2}}\right] \\
& -s_{W}^{2} \Pi_{\gamma \gamma}(0)-2 s_{W} c_{W} \Pi_{Z \gamma}(0),
\end{aligned}
$$

where $\Pi\left(p^{2}\right)$ are the contributions to the gauge bosons' self-energies from the BSM fields, at the (squared) energy scale $p^{2}$. $\alpha_{\mathrm{em}}$ (the fine-structure constant), $s_{W}=\sin \theta_{W}$ (the sinus of the Weinberg angle), $m_{W}$ and $m_{Z}$ take their experimental values as inferred within the SM. 
When fairly low mass states are present in a model, additional parameters $V, X$ and $W$ must be included to more precisely describe the impact of BSM physics on electroweak precision observables [111]. For example, $s_{W}^{2} /\left(s_{W}^{2}\right)_{\mathrm{SM}}$ is as function of $S, T$ and $X$; the ratio of decay widths $\Gamma(Z \rightarrow \nu \bar{\nu}) / \Gamma_{\mathrm{SM}}(Z \rightarrow \nu \bar{\nu})$ is a function of $T$ and $V$; the decay width ratio $\Gamma(W \rightarrow$ all $) / \Gamma_{\mathrm{SM}}(W \rightarrow$ all $)$ is a function of $S, T, U$ and $W$. In this work we concentrate only on the BSM contribution to the most relevant $S, T$ and $U$ parameters, whith ther $\mathrm{SM}$ values set to 0 at a top quark mass $m_{t}=173 \mathrm{GeV}$, higgs mass $m_{h}=126 \mathrm{GeV}$ and $\alpha_{\mathrm{em}}$ evaluated at the $m_{Z}$ scale [31].

In order to obtain the oblique correction induced by an inert $\mathrm{SU}(2)_{L}$ triplet scalar with hypercharge $Y=1$, a real $\mathrm{SU}(2)_{L}$ singlet scalar, and a doubly charged $\mathrm{SU}(2)_{L}$ singlet scalar, we derive the needed Feynman rules and evaluate the BSM contributions to the gauge boson self-energies $\Pi\left(p^{2}\right)$.

It turns out to be convenient to introduce the following function

$$
B_{5}\left(p^{2}, m_{i}^{2}, m_{j}^{2}\right)=4 B_{22}\left(p^{2}, m_{i}^{2}, m_{j}^{2}\right)-A\left(m_{i}^{2}\right)-A\left(m_{j}^{2}\right),
$$

where $A$ and $B_{22}$ are the Passarino-Veltman scalar functions given in [112] (for a modern approach to evaluate these functions see, e.g. [113], but note that this reference use an opposite sign convention for the scalar functions). All the contributions to eqs. (A.1)(A.3) can now be written in term of the BSM particle masses $\left(m_{\Delta^{++}}, m_{\Delta^{+}}, m_{A_{0}}, m_{S_{1}}\right.$, $m_{S_{2}}$ and $\rho^{++}$) through the $B_{5}$ function, and the singlet-triplet mixing angle $\alpha$. The BSM contributions to the gauge bosons' self-energies can be expressed as follows:

$$
\begin{aligned}
\Pi_{\gamma \gamma}\left(p^{2}\right)= & \frac{\alpha_{\mathrm{em}}}{4 \pi}\left[4 B_{5}\left(p^{2}, m_{\Delta^{++}}^{2}, m_{\Delta^{++}}^{2}\right)+B_{5}\left(p^{2}, m_{\Delta^{+}}^{2}, m_{\Delta^{+}}^{2}\right)\right]+\frac{\alpha_{\mathrm{em}}}{\pi} B_{5}\left(p^{2}, m_{\rho}^{2}, m_{\rho}^{2}\right) \\
\Pi_{Z \gamma}\left(p^{2}\right)= & \frac{\alpha_{\mathrm{em}}}{4 \pi} \frac{1}{s_{W} c_{W}}\left[2\left(c_{W}^{2}-s_{W}^{2}\right) B_{5}\left(p^{2}, m_{\Delta^{++}}^{2}, m_{\Delta^{++}}^{2}\right)-s_{W}^{2} B_{5}\left(p^{2}, m_{\Delta^{+}}^{2}, m_{\Delta^{+}}^{2}\right)\right] \\
& -\frac{\alpha_{\mathrm{em}}}{\pi} \frac{s_{W}}{c_{W}} B_{5}\left(p^{2}, m_{\rho}^{2}, m_{\rho}^{2}\right) \\
\Pi_{Z Z}\left(p^{2}\right)= & \frac{\alpha_{\mathrm{em}}}{4 \pi} \frac{1}{s_{W}^{2} c_{W}^{2}}\left[\left(c_{W}^{2}-s_{W}^{2}\right)^{2} B_{5}\left(p^{2}, m_{\Delta^{++}}^{2}, m_{\Delta^{++}}^{2}\right)-s_{W}^{4} B_{5}\left(p^{2}, m_{\Delta^{+}}^{2}, m_{\Delta^{+}}^{2}\right)\right. \\
& \left.+c_{\alpha}^{2} B_{5}\left(p^{2}, m_{S_{1}}^{2}, m_{A_{0}}^{2}\right)+s_{\alpha}^{2} B_{5}\left(p^{2}, m_{S_{2}}^{2}, m_{A_{0}}^{2}\right)\right]+\frac{\alpha_{\mathrm{em}}}{\pi} \frac{s_{W}^{2}}{c_{W}^{2}} B_{5}\left(p^{2}, m_{\rho}^{2}, m_{\rho}^{2}\right) \\
\Pi_{W W}\left(p^{2}\right)= & \frac{\alpha_{\mathrm{em}}}{4 \pi} \frac{1}{s_{W}^{2}}\left[B_{5}\left(p^{2}, m_{\Delta^{++}}^{2}, m_{\Delta^{+}}^{2}\right)+\frac{1}{2} B_{5}\left(p^{2}, m_{\Delta^{+}}^{2}, m_{A_{0}}^{2}\right)\right. \\
& \left.+\frac{c_{\alpha}^{2}}{2} B_{5}\left(p^{2}, m_{\Delta^{+}}^{2}, m_{S_{1}}^{2}\right)+\frac{s_{\alpha}^{2}}{2} B_{5}\left(p^{2}, m_{\Delta^{+}}^{2}, m_{S_{2}}^{2}\right)\right]
\end{aligned}
$$

where we recall that $s_{\alpha}=\sin \alpha$ sets the singlet (triplet) content of the $S_{1}\left(S_{2}\right)$ field. Additional analytical simplifications are possible, e.g. $B_{5}(0, m, m)=0$, and any remaining expressions and their derivatives are numerically evaluated with the FF package [114].

In order to derive constraints on BSM model parameters from the $S, T$ and $U$ measurements we evaluate the goodness-of-fit with the chi-square

$$
\chi^{2}=\sum_{x, y \in\{S, T, U\}}\left(x_{0}-x\right) V_{x y}^{-1}\left(y_{0}-y\right)
$$


From the particle data group [85] we take the experimental best-fit values to be $S_{0}=$ $-0.01 \pm 0.10, T_{0}=0.03 \pm 0.12, U_{0}=0.02 \pm 0.11$ with the errors denoting the 1-sigma variances $\sigma_{x}$, and $V_{x y}$ is the covariance matrix (or the error matrix) determined by

$$
V_{x y}=\sigma_{x} \sigma_{y} \rho_{x y}
$$

with the symmetric correlations matrix $\rho_{x y}$ given by the entries: $\rho_{x x}=1, \rho_{S T}=0.92$, $\rho_{S U}=-0.80, \rho_{T U}=-0.93$. A p-value of $5 \%$ is reached at $\chi^{2}=7.82$ (i.e. the $5 \%$ quantile for a $\chi^{2}$ distribution with 3 degrees of freedom). The best-fit models have $\chi^{2} \simeq 0$. For example, $\chi^{2} \simeq 0.032$ at the point $\lambda_{6}=1, s_{\alpha}=0.111, m_{\rho}=1 \mathrm{TeV}, m_{\Delta^{++}}=2.337 \mathrm{TeV}$ and $m_{\Delta^{+}}=2.363 \mathrm{TeV}$ (implying that $m_{S_{2}}=2.308 \mathrm{TeV}, m_{A_{0}}=2.390 \mathrm{TeV}, m_{S_{1}}=2.391 \mathrm{TeV}$ ) with the contribution $\{\Delta S \simeq 0.004, \Delta T \simeq 0.05, \Delta U \simeq 0\}$ in addition to the SM's $\left\{S_{\mathrm{SM}}=\right.$ $\left.T_{\mathrm{SM}}=U_{\mathrm{SM}}=0\right\}$. The regions in the $m_{\Delta^{++}}-m_{\Delta^{+}}$plane with p-values $>5 \%$ are shown in figure 7, where we made a scan over $50 \leq m_{\Delta^{++}} \leq 3000 \mathrm{GeV}, 50 \leq m_{\Delta^{+}} \leq 3000 \mathrm{GeV}$ and $-1 \leq \sin \alpha \leq 1$ for three fixed $\lambda_{6}=0.1,1$ and $4 \pi$, and for a fixed $m_{\rho}=1 \mathrm{TeV}$. We also constrained $S_{2}$ to have a positive mass and to be the lightest among the $\mathbb{Z}_{2}$-odd states, for it to be potentially viable DM candidate.

Open Access. This article is distributed under the terms of the Creative Commons Attribution License (CC-BY 4.0), which permits any use, distribution and reproduction in any medium, provided the original author(s) and source are credited.

\section{References}

[1] K.S. Babu and C.N. Leung, Classification of effective neutrino mass operators, Nucl. Phys. $B 619$ (2001) 667 [hep-ph/0106054] [INSPIRE].

[2] A. de Gouvêa and J. Jenkins, A survey of lepton number violation via effective operators, Phys. Rev. D 77 (2008) 013008 [arXiv:0708.1344] [INSPIRE].

[3] F. Bonnet, M. Hirsch, T. Ota and W. Winter, Systematic decomposition of the neutrinoless double beta decay operator, JHEP 03 (2013) 055 [Erratum ibid. 04 (2014) 090] [arXiv: 1212.3045] [INSPIRE].

[4] F.F. Deppisch, L. Graf, J. Harz and W.-C. Huang, Neutrinoless double beta decay and the baryon asymmetry of the universe, Phys. Rev. D 98 (2018) 055029 [arXiv:1711.10432] [inSPIRE].

[5] C.-S. Chen, C.Q. Geng and J.N. Ng, Unconventional neutrino mass generation, neutrinoless double beta decays, and collider phenomenology, Phys. Rev. D 75 (2007) 053004 [hep-ph/0610118] [INSPIRE].

[6] F. del Aguila, A. Aparici, S. Bhattacharya, A. Santamaria and J. Wudka, A realistic model of neutrino masses with a large neutrinoless double beta decay rate, JHEP 05 (2012) 133 [arXiv:1111.6960] [INSPIRE].

[7] F. del Aguila, A. Aparici, S. Bhattacharya, A. Santamaria and J. Wudka, Effective Lagrangian approach to neutrinoless double beta decay and neutrino masses, JHEP 06 (2012) 146 [arXiv: 1204.5986] [INSPIRE].

[8] M. Gustafsson, J.M. No and M.A. Rivera, Predictive model for radiatively induced neutrino masses and mixings with dark matter, Phys. Rev. Lett. 110 (2013) 211802 [Erratum ibid. 112 (2014) 259902] [arXiv:1212.4806] [INSPIRE]. 
[9] J. Alcaide, D. Das and A. Santamaria, A model of neutrino mass and dark matter with large neutrinoless double beta decay, JHEP 04 (2017) 049 [arXiv: 1701.01402] [INSPIRE].

[10] P.W. Angel, N.L. Rodd and R.R. Volkas, Origin of neutrino masses at the LHC: $\Delta L=2$ effective operators and their ultraviolet completions, Phys. Rev. D 87 (2013) 073007 [arXiv: 1212.6111] [INSPIRE].

[11] Y. Cai, J. Herrero-García, M.A. Schmidt, A. Vicente and R.R. Volkas, From the trees to the forest: a review of radiative neutrino mass models, Front. in Phys. 5 (2017) 63 [arXiv: 1706.08524] [INSPIRE].

[12] K.S. Babu, P.S.B. Dev, S. Jana and A. Thapa, Non-standard interactions in radiative neutrino mass models, JHEP 03 (2020) 006 [arXiv: 1907.09498] [INSPIRE].

[13] S. Weinberg, Baryon and lepton nonconserving processes, Phys. Rev. Lett. 43 (1979) 1566 [INSPIRE].

[14] M. Gustafsson, J.M. No and M.A. Rivera, Radiative neutrino mass generation linked to neutrino mixing and $0 \nu \beta \beta$-decay predictions, Phys. Rev. D 90 (2014) 013012 [arXiv:1402.0515] [INSPIRE].

[15] R. Cepedello, R.M. Fonseca and M. Hirsch, Systematic classification of three-loop realizations of the Weinberg operator, JHEP 10 (2018) 197 [Erratum ibid. 06 (2019) 034] [arXiv: 1807.00629] [INSPIRE].

[16] S.F. King, A. Merle and L. Panizzi, Effective theory of a doubly charged singlet scalar: complementarity of neutrino physics and the LHC, JHEP 11 (2014) 124 [arXiv: 1406.4137] [INSPIRE].

[17] T. Geib, S.F. King, A. Merle, J.M. No and L. Panizzi, Probing the origin of neutrino masses and mixings via doubly charged scalars: complementarity of the intensity and the energy frontiers, Phys. Rev. D 93 (2016) 073007 [arXiv: 1512.04391] [InSPIRE].

[18] L.-G. Jin, R. Tang and F. Zhang, A three-loop radiative neutrino mass model with dark matter, Phys. Lett. B 741 (2015) 163 [arXiv:1501.02020] [INSPIRE].

[19] W. Chao, G.-J. Ding, X.-G. He and M. Ramsey-Musolf, Scalar electroweak multiplet dark matter, JHEP 08 (2019) 058 [arXiv: 1812.07829] [INSPIRE].

[20] T.A. Chowdhury and S. Nasri, Gamma rays from Dark Matter Annihilation in Three-loop Radiative Neutrino Mass Generation Models, Phys. Lett. B 782 (2018) 215 [arXiv: 1802.05971] [INSPIRE].

[21] T.A. Chowdhury, S. Hassan, J. Hossain, S. Nasri and M.A. Shamim, Probing the Dark Matter of Three-loop Radiative Neutrino Mass Generation Model with the Cherenkov Telescope Array, arXiv:2008.06602 [INSPIRE].

[22] C.-S. Chen, K.L. McDonald and S. Nasri, A Class of Three-Loop Models with Neutrino Mass and Dark Matter, Phys. Lett. B 734 (2014) 388 [arXiv:1404.6033] [INSPIRE].

[23] J. Herrero-Garcia, M. Nebot, N. Rius and A. Santamaria, The Zee-Babu model revisited in the light of new data, Nucl. Phys. B 885 (2014) 542 [arXiv:1402.4491] [InSPIRE].

[24] A. Crivellin, M. Ghezzi, L. Panizzi, G.M. Pruna and A. Signer, Low- and high-energy phenomenology of a doubly charged scalar, Phys. Rev. D 99 (2019) 035004 [arXiv: 1807.10224] [INSPIRE].

[25] R. Cepedello, M. Hirsch, P. Rocha-Morán and A. Vicente, Minimal 3-loop neutrino mass models and charged lepton flavor violation, JHEP 08 (2020) 067 [arXiv:2005.00015] [INSPIRE]. 
[26] MEG collaboration, Search for the lepton flavour violating decay $\mu^{+} \rightarrow \mathrm{e}^{+} \gamma$ with the full dataset of the MEG experiment, Eur. Phys. J. C 76 (2016) 434 [arXiv:1605.05081] [INSPIRE].

[27] HFLAV collaboration, Averages of b-hadron, c-hadron, and $\tau$-lepton properties as of summer 2016, Eur. Phys. J. C 77 (2017) 895 [arXiv: 1612.07233] [InSPIRE].

[28] BABAR collaboration, Limits on $\tau$ lepton-flavor violating decays in three charged leptons, Phys. Rev. D 81 (2010) 111101 [arXiv:1002.4550] [inSPIRE].

[29] K. Hayasaka et al., Search for lepton flavor violating $\tau$ decays into three leptons with 719 million produced $\tau^{+} \tau^{-}$pairs, Phys. Lett. B $\mathbf{6 8 7}$ (2010) 139 [arXiv:1001.3221] [INSPIRE].

[30] SINDRUM collaboration, Search for the decay $\mu^{+} \rightarrow e^{+} e^{+} e^{-}$, Nucl. Phys. B 299 (1988) 1 [INSPIRE].

[31] Particle Data Group collaboration, Review of particle physics, Phys. Rev. D 98 (2018) 030001 [INSPIRE].

[32] M.E. Peskin and T. Takeuchi, Estimation of oblique electroweak corrections, Phys. Rev. D 46 (1992) 381 [INSPIRE].

[33] W. Grimus, L. Lavoura, O.M. Ogreid and P. Osland, The oblique parameters in multi-Higgs-doublet models, Nucl. Phys. B 801 (2008) 81 [arXiv:0802.4353] [InSPIRE].

[34] F. del Aguila, M. Chala, A. Santamaria and J. Wudka, Discriminating between lepton number violating scalars using events with four and three charged leptons at the LHC, Phys. Lett. B 725 (2013) 310 [arXiv:1305.3904] [inSPIRE].

[35] F. del Águila and M. Chala, LHC bounds on lepton number violation mediated by doubly and singly-charged scalars, JHEP 03 (2014) 027 [arXiv:1311.1510] [INSPIRE].

[36] S. Kanemura, K. Yagyu and H. Yokoya, First constraint on the mass of doubly-charged Higgs bosons in the same-sign diboson decay scenario at the LHC, Phys. Lett. B 726 (2013) 316 [arXiv: 1305.2383] [INSPIRE].

[37] S. Kanemura, M. Kikuchi, K. Yagyu and H. Yokoya, Bounds on the mass of doubly-charged Higgs bosons in the same-sign diboson decay scenario, Phys. Rev. D 90 (2014) 115018 [arXiv: 1407.6547] [INSPIRE].

[38] J. Alcaide, M. Chala and A. Santamaria, LHC signals of radiatively-induced neutrino masses and implications for the Zee-Babu model, Phys. Lett. B 779 (2018) 107 [arXiv: 1710.05885] [INSPIRE].

[39] ATLAS collaboration, Search for doubly-charged Higgs bosons in like-sign dilepton final states at $\sqrt{s}=7 \mathrm{TeV}$ with the ATLAS detector, Eur. Phys. J. C 72 (2012) 2244 [arXiv:1210.5070] [INSPIRE].

[40] ATLAS collaboration, Search for doubly-charged Higgs bosons in same-charge electron pair final states using proton-proton collisions at $\sqrt{s}=13 \mathrm{TeV}$ with the ATLAS detector, ATLAS-CONF-2016-051 (2016).

[41] K.S. Babu, Model of 'calculable' Majorana neutrino masses, Phys. Lett. B 203 (1988) 132 [INSPIRE].

[42] M. Nebot, J.F. Oliver, D. Palao and A. Santamaria, Prospects for the Zee-Babu model at the CERN LHC and low energy experiments, Phys. Rev. D 77 (2008) 093013 [arXiv:0711.0483] [INSPIRE]. 
[43] D. Schmidt, T. Schwetz and H. Zhang, Status of the Zee-Babu model for neutrino mass and possible tests at a like-sign linear collider, Nucl. Phys. B 885 (2014) 524 [arXiv: 1402.2251] [INSPIRE].

[44] K.S. Babu and C. Macesanu, Two loop neutrino mass generation and its experimental consequences, Phys. Rev. D 67 (2003) 073010 [hep-ph/0212058] [INSPIRE].

[45] C.-Q. Geng, D. Huang and L.-H. Tsai, Loop-induced neutrino masses: a case study, Phys. Rev. D 90 (2014) 113005 [arXiv: 1410.7606] [INSPIRE].

[46] A. Belyaev, G. Cacciapaglia, I.P. Ivanov, F. Rojas-Abatte and M. Thomas, Anatomy of the inert two Higgs doublet model in the light of the LHC and non-LHC dark matter searches, Phys. Rev. D 97 (2018) 035011 [arXiv:1612.00511] [INSPIRE].

[47] E. Ma, Verifiable radiative seesaw mechanism of neutrino mass and dark matter, Phys. Rev. D 73 (2006) 077301 [hep-ph/0601225] [INSPIRE].

[48] R. Barbieri, L.J. Hall and V.S. Rychkov, Improved naturalness with a heavy Higgs: an alternative road to LHC physics, Phys. Rev. D 74 (2006) 015007 [hep-ph/0603188] [INSPIRE].

[49] L. Lopez Honorez, E. Nezri, J.F. Oliver and M.H.G. Tytgat, The Inert Doublet Model: an archetype for dark matter, JCAP 02 (2007) 028 [hep-ph/0612275] [INSPIRE].

[50] M. Gustafsson, S. Rydbeck, L. Lopez-Honorez and E. Lundstrom, Status of the Inert Doublet Model and the role of multileptons at the LHC, Phys. Rev. D 86 (2012) 075019 [arXiv:1206.6316] [INSPIRE].

[51] A. Arhrib, Y.-L.S. Tsai, Q. Yuan and T.-C. Yuan, An updated analysis of inert Higgs doublet model in light of the recent results from LUX, PLANCK, AMS-02 and LHC, JCAP 06 (2014) 030 [arXiv: 1310.0358] [InSPIRE].

[52] A. Ilnicka, M. Krawczyk and T. Robens, Inert doublet model in light of LHC Run I and astrophysical data, Phys. Rev. D 93 (2016) 055026 [arXiv:1508.01671] [INSPIRE].

[53] C. Garcia-Cely, M. Gustafsson and A. Ibarra, Probing the inert doublet dark matter model with Cherenkov telescopes, JCAP 02 (2016) 043 [arXiv: 1512.02801] [INSPIRE].

[54] B. Eiteneuer, A. Goudelis and J. Heisig, The inert doublet model in the light of Fermi-LAT gamma-ray data: a global fit analysis, Eur. Phys. J. C 77 (2017) 624 [arXiv:1705.01458] [INSPIRE].

[55] E. Lundstrom, M. Gustafsson and J. Edsjo, The inert doublet model and LEP II Limits, Phys. Rev. D 79 (2009) 035013 [arXiv:0810.3924] [InSPIRE].

[56] G. Bélanger, B. Dumont, A. Goudelis, B. Herrmann, S. Kraml and D. Sengupta, Dilepton constraints in the inert doublet model from Run 1 of the LHC, Phys. Rev. D 91 (2015) 115011 [arXiv: 1503.07367] [INSPIRE].

[57] J. Kalinowski, W. Kotlarski, T. Robens, D. Sokolowska and A.F. Zarnecki, Benchmarking the inert doublet model for $e^{+} e^{-}$colliders, JHEP 12 (2018) 081 [arXiv:1809.07712] [INSPIRE].

[58] XENON collaboration, Dark matter search results from a one ton-year exposure of XENON1T, Phys. Rev. Lett. 121 (2018) 111302 [arXiv:1805.12562] [INSPIRE].

[59] M. Gustafsson, E. Lundstrom, L. Bergstrom and J. Edsjo, Significant gamma lines from inert Higgs dark matter, Phys. Rev. Lett. 99 (2007) 041301 [astro-ph/0703512] [InSPIRE].

[60] B. Dutta, G. Palacio, J.D. Ruiz-Alvarez and D. Restrepo, Vector boson fusion in the inert doublet model, Phys. Rev. D 97 (2018) 055045 [arXiv:1709.09796] [INSPIRE]. 
[61] A. Belyaev et al., Advancing LHC probes of dark matter from the inert two-Higgs-doublet model with the monojet signal, Phys. Rev. D 99 (2019) 015011 [arXiv:1809.00933] [INSPIRE].

[62] A. Arhrib, R. Benbrik and N. Gaur, $H \rightarrow \gamma \gamma$ in inert higgs doublet model, Phys. Rev. D 85 (2012) 095021 [arXiv:1201.2644] [INSPIRE].

[63] M. Krawczyk, D. Sokołowska, P. Swaczyna and B. Świeżewska, Higgs $\rightarrow \gamma \gamma, Z \gamma$ in the inert doublet model, Acta Phys. Polon. B 44 (2013) 2163 [arXiv: 1309.7880] [InSPIRE].

[64] V. Silveira and A. Zee, Scalar phantoms, Phys. Lett. B 161 (1985) 136 [inSPIRE].

[65] J. McDonald, Gauge singlet scalars as cold dark matter, Phys. Rev. D 50 (1994) 3637 [hep-ph/0702143] [INSPIRE].

[66] C.P. Burgess, M. Pospelov and T. ter Veldhuis, The minimal model of nonbaryonic dark matter: a singlet scalar, Nucl. Phys. B 619 (2001) 709 [hep-ph/0011335] [INSPIRE].

[67] L. Feng, S. Profumo and L. Ubaldi, Closing in on singlet scalar dark matter: LUX, invisible Higgs decays and gamma-ray lines, JHEP 03 (2015) 045 [arXiv:1412.1105] [INSPIRE].

[68] GAMBIT collaboration, Status of the scalar singlet dark matter model, Eur. Phys. J. C 77 (2017) 568 [arXiv: 1705.07931] [INSPIRE].

[69] M. Klasen, C.E. Yaguna and J.D. Ruiz-Alvarez, Electroweak corrections to the direct detection cross section of inert Higgs dark matter, Phys. Rev. D 87 (2013) 075025 [arXiv:1302.1657] [INSPIRE].

[70] O. Fischer and J.J. van der Bij, The scalar singlet-triplet dark matter model, JCAP 01 (2014) 032 [arXiv: 1311.1077] [INSPIRE].

[71] C. Cheung and D. Sanford, Simplified models of mixed dark matter, JCAP 02 (2014) 011 [arXiv: 1311.5896] [INSPIRE].

[72] M. Baumgart, I.Z. Rothstein and V. Vaidya, Constraints on galactic wino densities from gamma ray lines, JHEP 04 (2015) 106 [arXiv:1412.8698] [INSPIRE].

[73] M. Aoki, T. Toma and A. Vicente, Non-thermal production of minimal dark matter via right-handed neutrino decay, JCAP 09 (2015) 063 [arXiv:1507.01591] [INSPIRE].

[74] M. Cirelli, T. Hambye, P. Panci, F. Sala and M. Taoso, Gamma ray tests of minimal dark matter, JCAP 10 (2015) 026 [arXiv:1507.05519] [INSPIRE].

[75] C. Garcia-Cely, A. Ibarra, A.S. Lamperstorfer and M.H.G. Tytgat, Gamma-rays from heavy minimal dark matter, JCAP 10 (2015) 058 [arXiv:1507.05536] [INSPIRE].

[76] C. Garcia-Cely and A. Rivera, General calculation of the cross section for dark matter annihilations into two photons, JCAP 03 (2017) 054 [arXiv:1611.08029] [INSPIRE].

[77] D. Dercks and T. Robens, Constraining the inert doublet model using vector boson fusion, Eur. Phys. J. C 79 (2019) 924 [arXiv:1812.07913] [InSPIRE].

[78] J. Kalinowski, W. Kotlarski, T. Robens, D. Sokolowska and A.F. Zarnecki, The inert doublet model at current and future colliders, J. Phys. Conf. Ser. 1586 (2020) 012023 [arXiv: 1903.04456] [INSPIRE].

[79] L.M. Krauss, S. Nasri and M. Trodden, A model for neutrino masses and dark matter, Phys. Rev. D 67 (2003) 085002 [hep-ph/0210389] [INSPIRE].

[80] A. Ahriche and S. Nasri, Dark matter and strong electroweak phase transition in a radiative neutrino mass model, JCAP 07 (2013) 035 [arXiv:1304.2055] [INSPIRE]. 
[81] C. Guella, D. Cherigui, A. Ahriche, S. Nasri and R. Soualah, Probing radiative neutrino mass models with dilepton events at the LHC, Phys. Rev. D 93 (2016) 095022 [arXiv: 1601.04342] [INSPIRE].

[82] D. Cherigui, C. Guella, A. Ahriche and S. Nasri, Probing radiative neutrino mass models using trilepton channel at the LHC, Phys. Lett. B 762 (2016) 225 [arXiv:1605.03640] [INSPIRE].

[83] C.-Q. Geng, D. Huang and L.-H. Tsai, Comment on "A three-loop radiative neutrino mass model with dark matter" [Phys. Lett. B 741 (2015) 163], Phys. Lett. B $\mathbf{7 4 5}$ (2015) 56 [arXiv: 1504.05468] [INSPIRE].

[84] M. Gustafsson and M. Rivera, to appear.

[85] Particle Data Group collaboration, Review of particle physics, Prog. Theor. Exp. Phys. (2020) $083 \mathrm{C} 01$.

[86] P.J. Fox, R. Harnik, J. Kopp and Y. Tsai, LEP shines light on dark matter, Phys. Rev. D 84 (2011) 014028 [arXiv: 1103.0240] [INSPIRE].

[87] A. Freitas and S. Westhoff, Leptophilic dark matter in lepton interactions at LEP and ILC, JHEP 10 (2014) 116 [arXiv:1408.1959] [InSPIRE].

[88] K. Cheung and O. Seto, Phenomenology of TeV right-handed neutrino and the dark matter model, Phys. Rev. D 69 (2004) 113009 [hep-ph/0403003] [INSPIRE].

[89] M. Cirelli, N. Fornengo and A. Strumia, Minimal dark matter, Nucl. Phys. B 753 (2006) 178 [hep-ph/0512090] [INSPIRE].

[90] A. Dedes and D. Karamitros, Doublet-triplet fermionic dark matter, Phys. Rev. D 89 (2014) 115002 [arXiv:1403.7744] [INSPIRE].

[91] B. Pontecorvo, Inverse beta processes and nonconservation of lepton charge, Sov. Phys. JETP 7 (1958) 172 [Zh. Eksp. Teor. Fiz. 34 (1957) 247] [INSPIRE].

[92] Z. Maki, M. Nakagawa and S. Sakata, Remarks on the unified model of elementary particles, Prog. Theor. Phys. 28 (1962) 870 [inSPIRE].

[93] I. Esteban, M.C. Gonzalez-Garcia, A. Hernandez-Cabezudo, M. Maltoni and T. Schwetz, Global analysis of three-flavour neutrino oscillations: synergies and tensions in the determination of $\theta_{23}, \delta_{C P}$, and the mass ordering, JHEP 01 (2019) 106 [arXiv: 1811.05487] [INSPIRE].

[94] H. Pas, M. Hirsch, H.V. Klapdor-Kleingrothaus and S.G. Kovalenko, A superformula for neutrinoless double beta decay. 2. The Short range part, Phys. Lett. B 498 (2001) 35 [hep-ph/0008182] [INSPIRE].

[95] J. Bergstrom, A. Merle and T. Ohlsson, Constraining new physics with a positive or negative signal of neutrino-less double beta decay, JHEP 05 (2011) 122 [arXiv:1103.3015] [INSPIRE].

[96] M. Horoi and A. Neacsu, Towards an effective field theory approach to the neutrinoless double-beta decay, arXiv:1706.05391 [INSPIRE].

[97] M.J. Dolinski, A.W.P. Poon and W. Rodejohann, Neutrinoless double-beta decay: status and prospects, Ann. Rev. Nucl. Part. Sci. 69 (2019) 219 [arXiv: 1902.04097] [InSPIRE].

[98] GERDA collaboration, Improved limit on neutrinoless double- $\beta$ decay of ${ }^{76}$ Ge from GERDA Phase II, Phys. Rev. Lett. 120 (2018) 132503 [arXiv: 1803.11100] [InSPIRE]. 
[99] LEGEND collaboration, The Large Enriched Germanium Experiment for Neutrinoless Double Beta Decay (LEGEND), AIP Conf. Proc. 1894 (2017) 020027 [arXiv:1709.01980] [INSPIRE].

[100] KamLAND-Zen collaboration, Search for Majorana Neutrinos near the inverted mass hierarchy region with KamLAND-Zen, Phys. Rev. Lett. 117 (2016) 082503 [Addendum ibid. 117 (2016) 109903] [arXiv: 1605.02889] [INSPIRE].

[101] W. Dekens, J. de Vries, K. Fuyuto, E. Mereghetti and G. Zhou, Sterile neutrinos and neutrinoless double beta decay in effective field theory, JHEP 06 (2020) 097 [arXiv: 2002 .07182] [INSPIRE].

[102] NEXO collaboration, Sensitivity and discovery potential of nEXO to neutrinoless double beta decay, Phys. Rev. C 97 (2018) 065503 [arXiv:1710.05075] [InSPIRE].

[103] CUORE collaboration, First results from CUORE: a search for lepton number violation via $0 \nu \beta \beta$ decay of ${ }^{130}$ Te, Phys. Rev. Lett. 120 (2018) 132501 [arXiv: 1710.07988] [INSPIRE].

[104] D.R. Artusa et al., Enriched $\mathrm{TeO}_{2}$ bolometers with active particle discrimination: towards the CUPID experiment, Phys. Lett. B 767 (2017) 321 [arXiv:1610.03513] [InSPIRE].

[105] CUPID collaboration, CUPID pre-CDR, arXiv:1907.09376 [INSPIRE].

[106] A. Neacsu and M. Horoi, An effective method to accurately calculate the phase space factors for $\beta^{-} \beta^{-}$decay, Adv. High Energy Phys. 2016 (2016) 7486712 [arXiv:1510.00882] [INSPIRE].

[107] J. Suhonen and O. Civitarese, Weak-interaction and nuclear-structure aspects of nuclear double beta decay, Phys. Rept. 300 (1998) 123 [INSPIRE].

[108] D. Stefanik, R. Dvornicky, F. Simkovic and P. Vogel, Reexamining the light neutrino exchange mechanism of the $0 \nu \beta \beta$ decay with left-and right-handed leptonic and hadronic currents, Phys. Rev. C 92 (2015) 055502 [arXiv:1506.07145] [InSPIRE].

[109] J.J. Gomez-Cadenas et al., Sense and sensitivity of double beta decay experiments, JCAP 06 (2011) 007 [arXiv: 1010.5112] [INSPIRE].

[110] F.F. Deppisch, M. Hirsch and H. Pas, Neutrinoless double beta decay and physics beyond the standard model, J. Phys. G 39 (2012) 124007 [arXiv:1208.0727] [INSPIRE].

[111] I. Maksymyk, C.P. Burgess and D. London, Beyond S, T and U, Phys. Rev. D 50 (1994) 529 [hep-ph/9306267] [INSPIRE].

[112] G. Passarino and M.J.G. Veltman, One loop corrections for $e^{+} e^{-}$annihilation into $\mu^{+} \mu^{-}$ in the Weinberg model, Nucl. Phys. B 160 (1979) 151 [INSPIRE].

[113] J. Romao, Modern techniques for one-loop calculations, https://porthos.tecnico.ulisboa.pt/public/textos/one-loop.pdf.

[114] G.J. van Oldenborgh, FF: a package to evaluate one loop Feynman diagrams, Comput. Phys. Commun. 66 (1991) 1 [INSPIRE]. 